\title{
Randomization and Ambiguity Aversion
}

\author{
Shaowei Ke* Qi Zhang ${ }^{\dagger \ddagger}$
}

This Draft: January 21, 2020

\begin{abstract}
We propose a model of preferences in which the effect of randomization on ambiguity depends on how the unknown probability law is determined. We adopt the framework of Anscombe and Aumann (1963) and relax the axioms. In the resulting representation of the individual's preference, the individual has a collection of sets of priors $\mathcal{M}$. She believes that before she moves, nature has chosen an unknown scenario (a set of priors) from $\mathcal{M}$, and from that scenario, nature will choose a prior after she moves. The representation illustrates how randomization may partially eliminate the effect of ambiguity.
\end{abstract}

${ }^{*}$ Department of Economics, University of Michigan. Email: shaoweik@umich.edu.

${ }^{\dagger}$ ByteDance. Email: qizhang.berkeley@gmail.com.

†We thank the, three anonymous referees, Nemanja Antić, Tilman Börgers, Gabriel Carroll, Xiaoyu Cheng, David Dillenberger, Kfir Eliaz, Federico Echenique, Faruk Gul, Yoram Halevy, Edi Karni, Asen Kochov, Jihong Lee, Fabio Maccheroni, Yusufcan Masatlioglu, Bin Miao, Stephen Morris, Pietro Ortoleva, Wolfgang Pesendorfer, Philipp Sadowski, Kyoungwon Seo, Marciano Siniscalchi, Joel Sobel, Ran Spiegler, Lorenzo Stanca, Roee Teper, Chen Zhao, and Songfa Zhong for helpful comments. We also thank seminar and conference audiences at UMich, Xiamen University International Workshop on Experimental Economics, SAET, Columbia Conference on Economic Theory, UPitt, JHU, UPenn, D-TEA, and D-TEA China. 


\section{Introduction}

It is often assumed in economics that an individual has a unique subjective prior (probability measure) over states of the world, even though the true probability law-if one is already determined - is rarely provided. Ellsberg (1961) introduces intuitive thought experiments to show that, on the contrary, an individual usually faces ambiguity and is ambiguity-averse; that is, she does not have a unique prior and dislikes betting on an event without a unique subjective probability assessment. In one experiment, assuming that a correct bet yields $\$ 100$ and a wrong bet $\$ 0$, it is predicted that an individual facing a risky urn (that contains 50 red and 50 black balls) and an ambiguous urn (with 100 red and black balls, but in an unknown proportion) will prefer to bet on the color of a randomly drawn ball from the risky urn over that from the ambiguous urn. Nonetheless, fixing any urn, the individual will be indifferent between betting on either color of a randomly drawn ball. ${ }^{1}$ This prediction is confirmed by many subsequent studies.

In a seminal paper, Gilboa and Schmeidler (1989) introduce the maxmin expected utility (MEU) model to capture ambiguity aversion. In the model, the individual has multiple priors. To evaluate a choice object, such as a bet, she adopts the prior that minimizes its utility; to choose from multiple choice objects, she maximizes the minimum utility. ${ }^{2}$ The interpretation is that with multiple priors in mind, the individual is conservative and focuses on the worst-case scenario - or, equivalently, it is as if the individual believes that nature plays against her by choosing the worst probability law from a fixed set of laws.

The MEU model, or more generally the maxmin principle, has been useful in various areas of economics: contract theory, mechanism design, macroeconomics, finance, etc. ${ }^{3}$ Surpris-

\footnotetext{
${ }^{1}$ This implies that the individual must not have a unique prior, even if we consider (subjective) nonexpected utility models (see Machina and Schmeidler (1992)).

${ }^{2}$ In the previous experiment, suppose the individual has three priors about the composition of the ambiguous urn: The number of red balls is either 30,50 , or 70 . Then, to evaluate the bet on red (black) drawn from the ambiguous urn, she believes that there are only 30 red (black) balls.

${ }^{3}$ See, among many others, Hansen and Sargent (2010); Bergemann and Schlag (2011); Antić (2014); Easley, O'Hara, and Yang (2014); Di Tillio, Kos, and Messner (2017); and Carrasco, Farinha Luz, Kos, Messner, Monteiro, and Moreira (2018). In the MEU model, minimization is over the individual's multiple priors. The maxmin principle has also been adopted when minimization is based on regret, over multiple
} 
ingly, however, in these applications, it is usually unclear how a basic concept in economics, randomization/mixed strategies, should be modeled when an individual behaves according to the MEU model or any other maxmin criterion. ${ }^{4}$ Randomization is modeled differently in different applications or simply ruled out; sometimes a discussion of the difficulty of introducing randomization and various ways to model randomization is provided, but there is not yet a general principle that helps us better understand how randomization should be modeled in a given application. ${ }^{5}$

In this paper, we introduce a theory of randomization under ambiguity that may help address this issue. We focus on (randomization under) the MEU model, but the insights we derive may be applied to other ambiguity models and models with other maxmin criteria.

\subsection{How Should Randomization Eliminate the Effect of Ambigu- ity?}

Suppose there are two services, $A$ and $B$. An individual needs to purchase one of them. There are two states, $s_{A}$ and $s_{B}$. In state $s_{A}\left(s_{B}\right)$, service $A(B)$ yields payoff 1 and the other service yields 0 . Both services seem equally good to the individual, but similar to the urn experiment, the individual does not have a unique probability assessment of $s_{A}$ or $s_{B}$. Now, suppose the actual probability of $s_{A}$ and $s_{B}$ depends on some details of the services unknown to the individual. Consider two situations. In the first, those details are precisely stated in contracts, although the individual never plans to read them. In the second, service providers do not explain those details in contracts. If the individual chooses the services randomly, will randomization interact with ambiguity in any way in either situation?

To understand the answer to this question, we first return to the urn experiment, in which the individual is indifferent between betting on red and black being drawn from the mechanisms, over multiple production technologies, over opponents' preferences, etc.

${ }^{4}$ In the literature on ambiguity, two types of mixture operations have been studied. Here, by randomization, we mean the mixture operation implemented before the state is revealed to the individual. In Section 2.1, we will introduce the other type of mixture operation, state-wise randomization.

${ }^{5}$ See Section 5 for more details. 
ambiguous urn. Raiffa (1961) points out that randomization renders ambiguity irrelevant. Suppose the individual tosses a fair coin, and bets on red being drawn from the ambiguous urn if heads and black if tails. Then, no matter what the number of red/black balls is in the ambiguous urn and which color is drawn, the individual will bet on red and black with equal probability; that is, she always receives a 50-50 lottery between $\$ 100$ and $\$ 0$ (see the right-hand side of Figure 1), which is what she will receive if she bets on red/black being drawn from the risky urn. ${ }^{6}$ Therefore, if randomization is perceived in this way, ambiguity becomes irrelevant.

However, randomization can be viewed in other ways. A popular alternative view (the left-hand side of Figure 1) is as follows: Regardless of the outcome of the coin toss, the individual must bet on either red or black being drawn from the ambiguous urn; that is, after the coin toss, she always ends up with a bet from the ambiguous urn that she dislikes in the first place. As Saito (2015) argues, if ambiguity is perceived in this way, its effect should be unaffected by randomization. To date, in applications that apply the maxmin principle, if mixed strategies are not ruled out, either the first view or the second is adopted. ${ }^{7}$
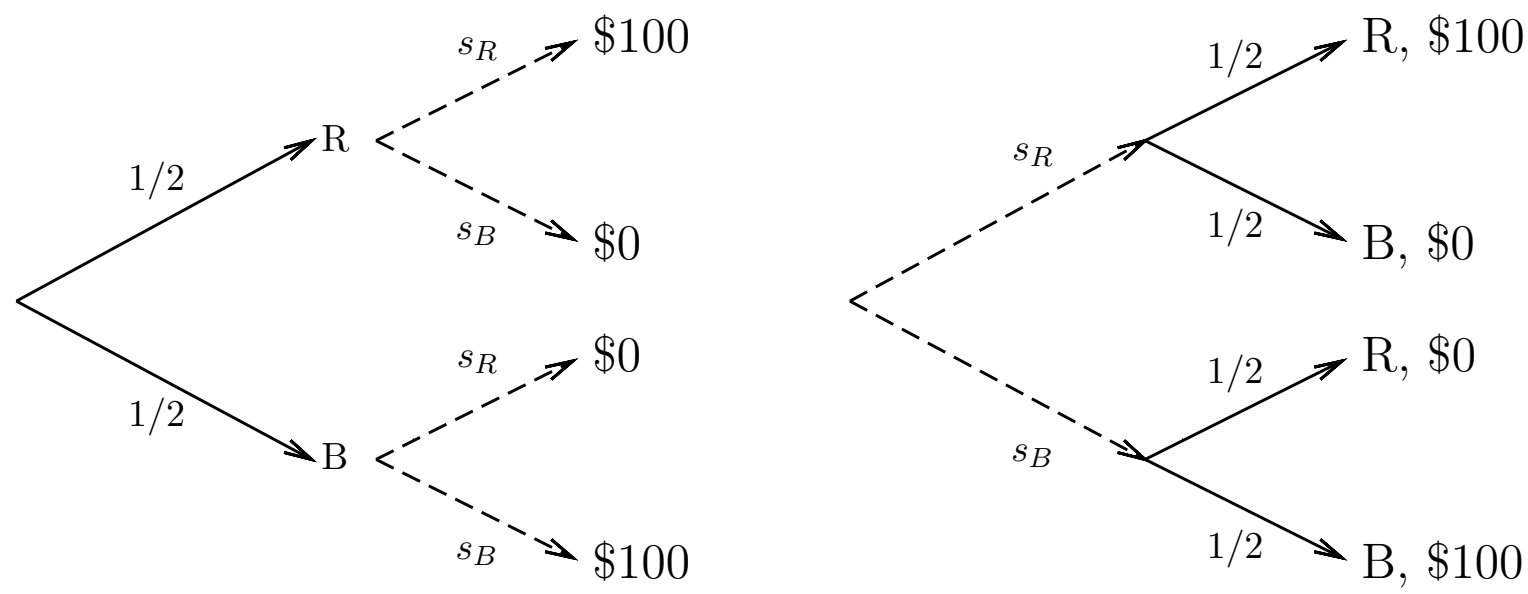

Figure 1: There are two states, in which $s_{R}\left(s_{B}\right)$ means that red (black) is drawn from the ambiguous urn. Randomization is represented by solid lines, with " $R$ " ("B") representing betting on red (black). The number along a solid line indicates the objective probability of betting on the corresponding color.

\footnotetext{
${ }^{6}$ The right-hand side of Figure 1 is in fact a simplified version of this idea. See Figure 3.

${ }^{7}$ See Section 5 for a more detailed discussion.
} 
A few papers have noted that the main difference between the two views lies in subjective timing. ${ }^{8}$ This is easier to explain under the MEU model. Suppose the individual believes that the number of red balls in the ambiguous urn is either 30 or 70 . Recall that an as-if interpretation of the MEU model is that the individual believes that nature plays against her. Does she believe that she randomizes before nature moves or after? If she believes that she moves first, nature can choose the probability law based on the outcome of the coin toss: If heads (betting on red), the number of red balls will be 30; otherwise, it is 70 . Either way, the individual's bet will be correct with only $30 \%$ chance. This is consistent with the second view: Regardless of the outcome of the coin toss, the individual is affected by ambiguity as in the case without the coin.

This is not true, however, if the individual believes that nature moves first. If nature moves first, although the number of red balls is unknown, it cannot depend on the outcome of the coin toss. Suppose the fixed number of red balls is 30. If heads, the individual's bet will be correct with $30 \%$ chance; otherwise, it will be correct with $70 \%$ chance. Overall, the individual's bet will be correct with $50 \%$ chance. The same applies if the fixed number of red balls is 70. Therefore, this is consistent with the first view: Fixing an arbitrary number of red balls, the coin toss always gives the individual a 50-50 lottery between $\$ 100$ and $\$ 0$. Thus, the interaction between randomization and ambiguity should depend on the individual's belief about how the unknown probability law is determined.

Now, return to the service example. In the first situation, before the individual randomizes, the contracts have pinned down the probability of $s_{A}$ and $s_{B}$, although the probability is unknown to the individual. This is similar to the case in which the number of red balls does not depend on the outcome of the coin toss, and hence the view that randomization renders ambiguity irrelevant is more suitable. In the second situation without contracts, it seems more plausible that the effect of ambiguity is unaffected by randomization: The individual

\footnotetext{
${ }^{8}$ Among others, see Epstein, Marinacci, and Seo (2007); Bade (2015); Saito (2015); Baillon, Halevy, and Li (2019); and Oechssler, Rau, and Roomets (2019). Note that subjective timing does not have to be identical to objective timing (if there is any).
} 
may worry that after randomization and choosing a service, the details of the service will still be manipulated in a way against her.

In the service example, first, there is a natural objective timing of when the probability law is determined. This is often not the case, and even if there is a natural objective timing, the individual is free to hold a different view. Second, the timing is extreme, in the sense that the probability law is completely pinned down either before randomization or after. Again, this rarely happens in practice. For example, in the first situation of the service example, if contracts use words that can be interpreted in many ways, and "The service provider reserves all rights for final explanation" is added to both contracts, how does it change the individual's view of randomization?

Similarly, consider a stylized investment example in which the individual can either long or short a firm. Suppose she believes that the probability that the firm succeeds depends on some unknown long-term factor that has been determined and some unknown short-term factor that will be determined after she makes a decision. How should randomization interact with ambiguity?

\subsection{Preview of Results}

To study randomization under ambiguity, we adopt the choice domain of Anscombe and Aumann (1963) (henceforth AA) and analyze an individual's preference. ${ }^{9}$ The domain consists of lotteries over acts (lotteries for short henceforth; see Figure 2). A lottery is a probability measure (mixed strategy) over acts. An act assigns to each state of the world a prize. A prize is a probability measure over consequences. Note that this domain does not require that we describe objectively how the probability law is determined; this will be subjective and revealed from the individual's preference.

We incorporate ideas from Gilboa and Schmeidler (1989) and first- and second-order

\footnotetext{
${ }^{9}$ We use the original AA choice domain that has two types of mixture operations. In many papers using the AA choice domain, only one mixture operation, which will be called state-wise randomization in our paper, is involved. See Section 5 for more details.
} 


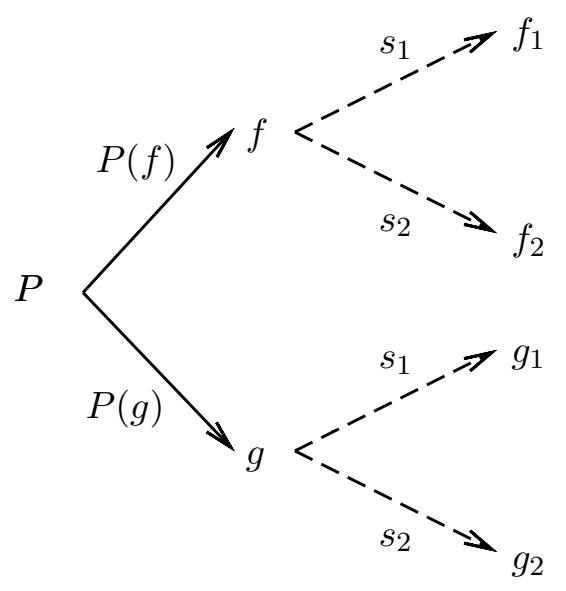

Figure 2: A lottery $P$ that yields act $f$ with probability $P(f)$ and act $g$ with $P(g)$, in which $f_{i}\left(g_{i}\right)$ is the prize associated with state $s_{i}$ by $f(g)$.

stochastic dominance to relax AA's axioms. Our main theorem characterizes the following representation of the individual's preference over lotteries, the double maxmin expected utility (DMEU) representation: For each lottery $P$,

$$
W(P)=\min _{M \in \mathcal{M}} \int_{\mathcal{F}}\left(\min _{\mu \in M} \int_{\mathcal{S}} u(f) d \mu\right) d P,
$$

in which each $M \in \mathcal{M}$ is a set of priors, and $u(f(s))$ is the utility of the prize that an act $f$ assigns to state $s$. Each $M \in \mathcal{M}$ is called a scenario. The individual believes that before she randomizes, nature, which plays against her, has chosen a scenario (although unknown to her) from $\mathcal{M}$. After she randomizes, nature will choose a prior $\mu$ from that scenario.

Figures 3-5 illustrate how the DMEU representation describes the individual's belief about the determination of the unknown probability law and about the interaction between randomization and ambiguity. Figure 3 depicts the urn experiment assuming that the individual believes that the number of red balls is determined before she randomizes and hence randomization eliminates the effect of ambiguity. This DMEU representation has two scenarios and each scenario has only one probability law. Figure 4 depicts the urn experiment assuming that the individual believes that the number of red balls is determined after she randomizes and hence the effect of ambiguity is unaffected by randomization. This DMEU 


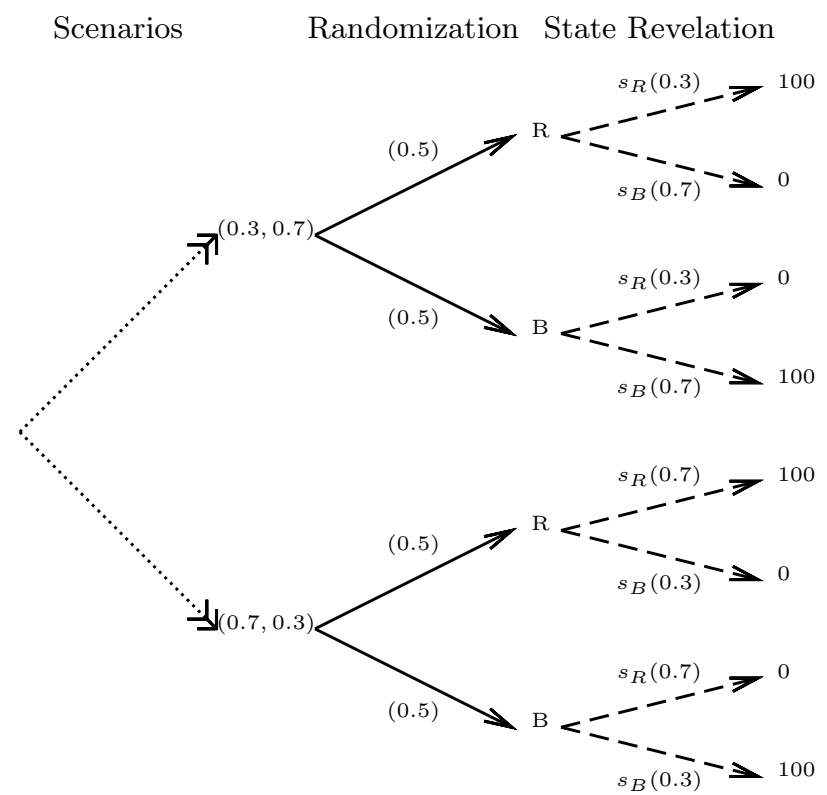

$$
\begin{array}{cc}
\text { use the } & \text { use the only prior } \\
\text { worst scenario } & \text { in each scenario }
\end{array}
$$

Figure 3: There are two scenarios. For example, $(0.3,0.7)$ is the scenario with 30 red balls and 70 black balls in the ambiguous urn. There is only one prior in each scenario. In state $s_{R}\left(s_{B}\right)$, the ball drawn is red (black). In each scenario, randomization and state revelation constitute a compound lottery that yields $\$ 100$ and $\$ 0$ with equal probability.

representation has only one scenario and that scenario has multiple probability laws.

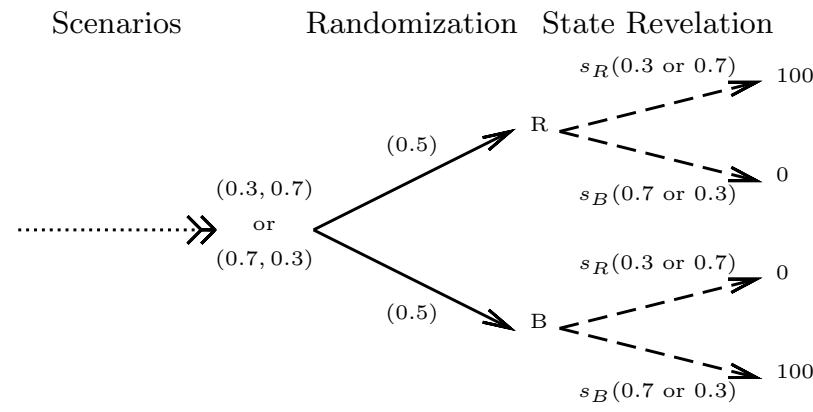

$$
\begin{array}{cc}
\text { use the } & \text { use the worst prior } \\
\text { only scenario } & \text { in each scenario }
\end{array}
$$

Figure 4: The only scenario contains two priors (either 30 or 70 red balls in the ambiguous urn). The worst prior within the scenario depends on the realization of randomization.

Figure 5 is the investment example, in which the probability that the firm succeeds depends on some unknown long-term and short-term factors. This example shows that 
randomization may partially eliminate the effect of ambiguity — it helps eliminate the effect of ambiguity across scenarios, but not across probability laws within a scenario. The individual believes that one unknown scenario in $\mathcal{M}=\left\{M_{G}, M_{N}, M_{B}\right\}$ has occured before she chooses. Scenarios $M_{G}$ (good long-term factor) and $M_{B}$ (bad long-term factor) are singletons - the long-term factor alone determines the probability law. In scenario $M_{N}$, how likely the firm is to succeed also depends on the short-term factor, which is not yet determined. In this case, randomization eliminates the effect of ambiguity across scenarios $M_{G}$ and $M_{B}$, but not within $M_{N} \cdot^{10}$

Next, we discuss the related literature; additional discussion can be found in Section 5. The closest paper to ours is Saito (2015). Saito also studies an axiomatic model in which the ambiguity-averse individual may have a preference for randomization. His model uses a convex combination to combine the two extreme timing beliefs - the belief that nature moves completely before the individual randomizes and the belief that nature moves completely after. We will show that his model is a special case of ours. In addition to models of ambiguity aversion, there are other models of preference for randomization under different choice domains with different motivations, such as Machina (1985); Cerreia-Vioglio, Dillenberger, and Ortoleva (2015); Fudenberg, Iijima, and Strzalecki (2015); and Cerreia-Vioglio, Dillenberger, Ortoleva, and Riella (2019), among others.

Several papers have also examined representations of preferences that involve collections of sets of priors. Lehrer and Teper (2011) study a representation called the multiple multiplepriors representation, which allows for violations of completeness and transitivity. Frick, Iijima, and Le Yaouanq (2019) propose the Boolean expected utility representation, in which the belief the individual uses to evaluate an act is the most pessimistic prior from the most optimistic set of priors.

Empirical studies have examined whether individuals strictly prefer to randomize. For example, Dominiak and Schnedler (2011); Agranov and Ortoleva (2017); Dwenger, Kübler,

\footnotetext{
${ }^{10} \mathrm{~A}$ more detailed discussion follows Theorem 2.
} 


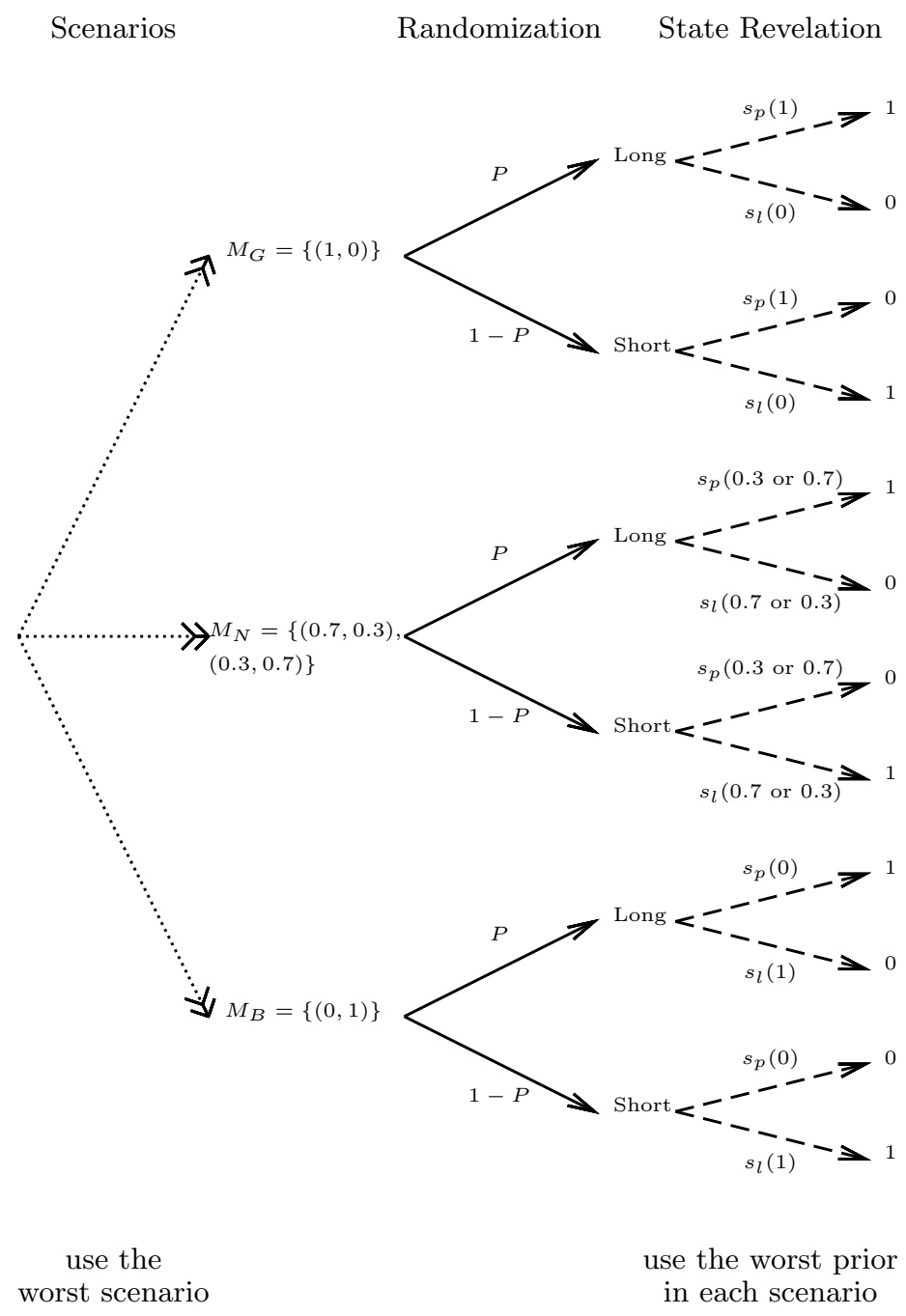

Figure 5: In the good scenario $M_{G}$, the firm will profit (state $s_{p}$ ) with probability 1 . In the bad scenario $M_{B}$, the firm will profit with probability 0 . In the neutral scenario $M_{N}$, the probability that the firm profits is either 0.3 or 0.7 , which will be determined after randomization.

and Weizsäcker (2018); and Oechssler et al. (2019). Many of them find a nonnegligible number of individuals who strictly prefer to randomize, but some do not. This is consistent with our theory: The individual may strictly prefer to randomize in some choice problems but not in others, and how desirable randomization appears to her depends on her timing beliefs.

Preference for randomization is also an important topic in preference elicitation in experiments. Indeed, our main idea that the individual's evaluation of a lottery depends on 
her belief about how the unknown probability law is determined has also appeared in the research on random incentive mechanisms. Bade (2015) and Baillon et al. (2019) argue that ambiguity-averse participants may use the randomization device to hedge, and thus the incentive compatibility of the random incentive mechanism may be affected. Kuzmics (2017) also points out the difficulty of preference elicitation when individuals face ambiguity: Individuals with nonsubjective-expected-utility preferences may end up behaving as if they are subjective-expected-utility maximizers.

Finally, many papers have studied ambiguity and robustness in different areas of economics. As mentioned earlier, how mixed strategies should be introduced and evaluated has been an issue. Our results offer some new insights into this issue. We leave the detailed discussion to the end of the paper.

The paper proceeds as follows. Section 2 introduces the choice domain, mixture operations, and a variant of AA's characterization of the subjective expected utility representation. Section 3 presents the main axioms, the DMEU representation, and the main results. Section 4 examines three special cases of the DMEU. Section 5 discusses the related literature.

\section{Preliminaries}

The set of consequences is a compact Polish space $\mathcal{X}{ }^{11}$ Let $\Delta(\mathcal{X})$ be the set of Borel probability measures on $\mathcal{X}$, endowed with the topology of weak convergence. Elements of $\Delta(\mathcal{X})$ are called prizes. Let $\mathcal{S}=\left\{s_{1}, \ldots, s_{n}\right\}$ be a finite set of states. An act $f: \mathcal{S} \rightarrow \Delta(\mathcal{X})$ is a function that assigns a prize to each state. For each state $s_{i}$, we write $f_{i}$ instead of $f\left(s_{i}\right)$ for simplicity. Let $\mathcal{F}$ denote the set of all acts, endowed with the product topology. Let $\Delta(\mathcal{F})$, the set of Borel probability measures on $\mathcal{F}$, denote the set of all lotteries, endowed with the topology of weak convergence. Henceforth, when we say a set of acts, we mean a Borel measurable subset of $\mathcal{F}$. The support of a lottery $P \in \Delta(\mathcal{F})$, denoted by $\operatorname{supp}(P)$, is the smallest closed set of acts $F \subset \mathcal{F}$ such that $P(F)=1$. The individual has a binary

\footnotetext{
${ }^{11} \mathrm{~A}$ Polish space is a complete separable metric space.
} 
relation/preference $\succsim$ on $\Delta(\mathcal{F})$. We denote the asymmetric part of $\succsim$ by $\succ$ and the symmetric part by $\sim$.

A lottery represents randomization over acts. Following randomization, the individual receives an act. Next, a state is revealed to the individual, after which the act assigns the prize associated with the realized state to the individual. Finally, as the prize's risk resolves, the individual receives a consequence (see Figure 2).

Lotteries are denoted by $P, Q, R$, acts are denoted by $f, g, h$, and prizes are denoted by $p, q, r$. A degenerate lottery that assigns probability 1 to an act $f$ is identified with $f$. If an act yields the same prize $p$ in all states, the act is called a constant act, and is identified with $p$. If a prize assigns probability 1 to a consequence $x$, we denote the prize by $\delta_{x}$.

We assume throughout the paper that $\succsim$ is nontrivial: There exist some lotteries $P$ and $Q$ such that $P \succ Q$.

\subsection{Randomization and State-wise Randomization}

We distinguish between two kinds of mixture operations. First, the randomization between lotteries $P$ and $Q$ with probability $\alpha \in[0,1]$, denoted by $\alpha P+(1-\alpha) Q$, is a lottery that assigns probability $\alpha P(F)+(1-\alpha) Q(F)$ to each set of acts $F \subset \mathcal{F}$. The second kind of mixture operation is state by state and for acts. The state-wise randomization between acts $f$ and $g$ with probability $\alpha \in[0,1]$, denoted by $\alpha f+_{s w}(1-\alpha) g$, is an act that assigns the prize $\alpha f_{i}+(1-\alpha) g_{i}$ to each state $s_{i}$ (see Figure 6$) .{ }^{12}$

The difference between the two mixture operations is obvious, but one useful way to understand it is to consider randomization as a mixture performed before the state is revealed to the individual, and state-wise randomization as a mixture performed after the state is revealed. In the urn experiment, before a ball is drawn, the individual tosses a fair coin and bets on red being drawn from the ambiguous urn if heads and black if tails. This is

\footnotetext{
${ }^{12}$ Here, $\alpha f_{i}+(1-\alpha) g_{i}$ is the standard mixture between prizes (probability measures over consequences) used in expected utility theory. With an abuse of notation, this mixture is also denoted by "+." In most other places in the paper, however, "+" represents randomization between lotteries.
} 


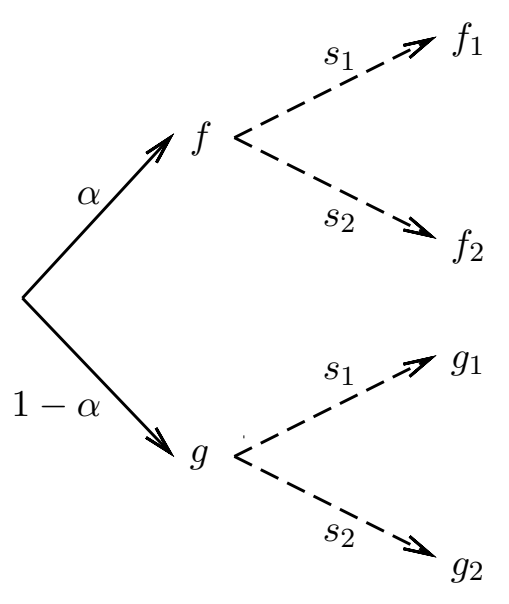

Randomization

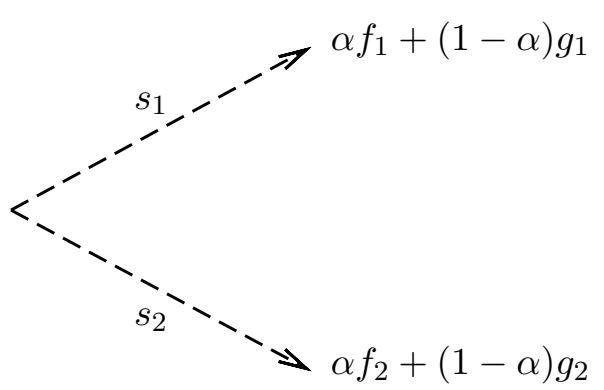

State-wise Randomization

Figure 6: Suppose there are two states: $s_{1}$ and $s_{2}$. The left-hand lottery is the randomization with probability $\alpha$ between acts (degenerate lotteries) $f$ and $g$. The right-hand act is the state-wise randomization with probability $\alpha$ between acts $f$ and $g$.

randomization and is done before the color of the drawn ball is revealed to the individual. As discussed in Section 1, whether such randomization eliminates the effect of ambiguity depends on the individual's subjective timing belief.

In contrast, suppose the individual first observes the color of the ball drawn, and then tosses the coin and bets accordingly. This will generate an act that yields a 50-50 lottery between $\$ 100$ and $\$ 0$ in all states, $s_{R}$ (red is drawn) and $s_{B}$ (black is drawn), which is exactly the 50-50 state-wise randomization between betting on red and black (see also the right-hand side of Figures 1 and 6 ). Since the prizes are identical in all states, this 50-50 state-wise randomization eliminates the effect of ambiguity, regardless of the individual's belief about how the unknown probability law is determined. To put it differently, because the mixture happens after the state is revealed, nature must have determined the probability law, in which case the individual's timing belief no longer matters.

From the above discussion, it can also be seen that under the same mixture probability, state-wise randomization should be (weakly) more effective in eliminating the effect of ambiguity than randomization. Intuitively, under state-wise randomization, nature always moves before the mixture, and hence it is (weakly) harder for nature to play against the individual 
than under randomization.

\subsection{Subjective Expected Utility}

AA provide a characterization of the subjective expected utility (SEU) representation. We introduce a variant that will be useful in motivating our axioms in Section 3 . The first four axioms are restatements of AA's axioms and assumptions using our terminology and notation.

Axiom 1 (Weak Order) The preference $\succsim$ is complete and transitive.

Axiom 2 (Continuity) For any $P \in \Delta(\mathcal{F}),\{Q \in \Delta(\mathcal{F}): Q \succsim P\}$ and $\{Q \in \Delta(\mathcal{F}): P \succsim Q\}$ are closed.

The first two axioms are basic. The next three axioms are the ones we want to relax later.

Axiom 3 (Independence) For any $P, Q, R \in \Delta(\mathcal{F})$ and $\alpha \in(0,1), P \succsim Q$ if and only if $\alpha P+(1-\alpha) R \succsim \alpha Q+(1-\alpha) R$.

Axiom 4 (State-wise Independence) For any $f, g, h \in \mathcal{F}$ and $\alpha \in(0,1), f \succsim g$ if and only if $\alpha f+_{s w}(1-\alpha) h \succsim \alpha g+_{s w}(1-\alpha) h$.

To state the last axiom, we need the following notation. For each lottery $P$, let $f^{P}$ denote the act such that

$$
f_{i}^{P}(X)=\int_{\mathcal{F}} f_{i}(X) d P
$$

for any $i \in\{1, \ldots, n\}$ and measurable set of consequences $X \subset \mathcal{X}$. By construction, the lottery $P$ is converted into an act $f^{P}$ state by state via reduction of compound lotteries. For example, suppose the support of $P$ is finite. Fixing any state $s_{i}, f_{i}^{P}$ is the mixture of prizes weighted by $P, \sum_{f \in \operatorname{supp}(P)} P(f) \cdot f_{i}$, which is a standard compound lottery in expected utility theory. To put it differently, if we convert all randomization in $P$ into state-wise randomization, we obtain $f^{P}$. 
Axiom 5 (Strong Dominance) For any $P, Q \in \Delta(\mathcal{F})$, if $f_{i}^{P} \succsim f_{i}^{Q}$ for any $i$, then $P \succsim Q$.

This axiom combines AA's two axioms, Monotonicity in Prizes and Reversal of Order in Compound Lotteries. Monotonicity in Prizes requires that for any two acts $f$ and $g$, if $f_{i} \succsim g_{i}$ for every $i, f \succsim g$. Reversal of Order in Compound Lotteries requires that $f^{P} \sim P$. Therefore, Strong Dominance requires that the individual be indifferent between randomization and state-wise randomization. The lemma below emphasizes this, and illustrates some basic relation between Independence, State-wise Independence, and Strong Dominance. All omitted proofs can be found in the Appendix.

Lemma 1 Suppose $\succsim$ satisfies Weak Order. The following statements are true:

1. For any $P, Q \in \Delta(\mathcal{F})$ and $\alpha \in[0,1]$, Strong Dominance implies that $\alpha P+(1-\alpha) Q \sim$ $f^{\alpha P+(1-\alpha) Q} \sim \alpha f^{P}+(1-\alpha) f^{Q} \sim \alpha f^{P}+{ }_{s w}(1-\alpha) f^{Q}$

2. Under Strong Dominance, Independence is equivalent to State-wise Independence.

The axioms above characterize the SEU representation. A function $u: \Delta(\mathcal{X}) \rightarrow \mathbb{R}$ is an expected utility function if for any prize $p$,

$$
u(p)=\int_{\mathcal{X}} u\left(\delta_{x}\right) d p .
$$

Let $\Delta(\mathcal{S})$ denote the set of probability measures on $\mathcal{S}$.

Definition 1 The individual's preference $\succsim$ has an SEU representation if there exists a continuous expected utility function $u: \Delta(\mathcal{X}) \rightarrow \mathbb{R}$ and $\mu \in \Delta(\mathcal{S})$ such that the following function $W$ represents $\succsim:$

$$
W(P)=\int_{\mathcal{F}}\left(\int_{\mathcal{S}} u(f) d \mu\right) d P .
$$

Since we assume that $\succsim$ is nontrivial, implicitly in the definition above, $W$ is not constant. We will not repeat this for our other representations, but similar implicit assumptions apply to them as well. 
When $\succsim$ has an SEU representation, the individual is indifferent between randomization and state-wise randomization, which is reflected in the following equations:

$$
W(P)=\int_{\mathcal{F}}\left(\int_{\mathcal{S}} u(f) d \mu\right) d P=\int_{\mathcal{S}}\left(\int_{\mathcal{F}} u(f) d P\right) d \mu=\int_{\mathcal{S}} u\left(f^{P}\right) d \mu=W\left(f^{P}\right) .
$$

We say that an SEU representation is unique if in (2), $u$ is unique up to a positive affine transformation and $\mu$ is unique. The theorem below restates AA's main representation result.

Theorem 1 (Anscombe and Aumann (1963)) The individual's preference $\succsim$ has an SEU representation if and only if it satisfies Weak Order, Continuity, Independence, State-wise Independence, and Strong Dominance. Furthermore, the SEU representation is unique.

Because Strong Dominance holds, Lemma 1 implies that one of the two axioms, Independence or State-wise Independence, is redundant. Stating the theorem in this way, however, helps us motivate the relaxations we introduce below.

\section{Axioms and the Representation}

Three axioms will be relaxed. Observing that state-wise randomization may render ambiguity irrelevant because of hedging (as discussed in Section 2.1), Gilboa and Schmeidler (1989) propose a relaxation of their independence assumption, which is State-wise Independence in our setting, to capture this. They allow the individual's preference to be convex so that she may strictly prefer state-wise randomization; meanwhile, constant acts should continue to satisfy the independence assumption, because constant acts do not provide hedging opportunities. We relax State-wise Independence in the same way.

We also want to relax Independence so that the individual may strictly prefer randomization. In the urn experiment, if the individual agrees with Raiffa's (1961) argument - or, in the service example, if the details of the services are precisely stated in contracts beforehandrandomization can render ambiguity irrelevant. In the investment example in Figure 5, 
randomization partially eliminates the effect of ambiguity, which also violates Independence. We relax Independence following the way Gilboa and Schmeidler (1989) relax State-wise Independence.

We say that a lottery $P$ preserves Independence if for any lotteries $Q, R$ and $\alpha \in(0,1)$, $Q \succsim R$ if and only if $\alpha Q+(1-\alpha) P \succsim \alpha R+(1-\alpha) P$. We say that an act $f$ preserves Statewise Independence if for any acts $g, h$ and $\alpha \in(0,1), g \succsim h$ if and only if $\alpha g+_{s w}(1-\alpha) f \succsim$ $\alpha h+{ }_{s w}(1-\alpha) f$

Axiom 6 (Preference for Randomization)

(i) For any $P, Q \in \Delta(\mathcal{F})$ and $\alpha \in[0,1], P \succsim Q$ implies $\alpha P+(1-\alpha) Q \succsim Q$;

(ii) Every constant act preserves Independence.

Axiom 7 (Preference for State-wise Randomization)

(i) For any $f, g \in \mathcal{F}$ and $\alpha \in[0,1], f \succsim g$ implies $\alpha f+{ }_{s w}(1-\alpha) g \succsim g$;

(ii) Every constant act preserves State-wise Independence.

Next, we replace Strong Dominance with three weaker axioms. Strong Dominance requires that the individual be indifferent between randomization and state-wise randomization, but as discussed earlier, the individual may not agree that randomization is as useful in eliminating the effect of ambiguity as state-wise randomization. For instance, suppose an individual believes that the effect of ambiguity is unaffected by randomization in the urn experiment. Since the 50-50 state-wise randomization renders ambiguity irrelevant in the urn experiment (as discussed in Section 2.1), this individual must have viewed randomization and state-wise randomization differently. Hence, Strong Dominance is violated. ${ }^{13}$

\footnotetext{
${ }^{13}$ An individual can simultaneously violate Independence, State-wise Independence, and Strong Dominance. To see this, consider an individual who believes that randomization eliminates the effect of ambiguity in the service example with precise contracts (which suggests that Independence is violated), and at the same time believes that ambiguity is unaffected by randomization in the urn experiment. Since the 50-50 state-wise randomization renders ambiguity irrelevant in the urn experiment, both State-wise Independence and Strong Dominance are violated.
} 
Indifference between the two types of mixture operations arises because to compare two lotteries, $P$ and $Q$, Strong Dominance first converts them to two acts, state by state, via reduction of compound lotteries (see (1)). The conversion turns randomization into state-wise randomization and implicitly identifies the two types of mixture operations. Therefore, a natural weakening is to compare $P$ and $Q$ without the conversion. We compare them realization by realization and state by state. The relation between realization-by-realization comparison of lotteries and first-order stochastic dominance is well known. Following Lehmann (1955), we say that a set of acts $F \subset \mathcal{F}$ is increasing if, whenever $f_{i} \succsim g_{i}$ for any $i$ and $g \in F$, we have $f \in F$.

Definition 2 We say that $P$ first-order stochastically dominates $Q$ if for any increasing set of acts $F \subset \mathcal{F}, P(F) \geq Q(F)$.

For any two acts $f$ and $g$, we think of $f$ as an improvement of $g$ if $f_{i} \succsim g_{i}$ for every $i \in\{1, \ldots, n\}$. The condition in the above definition implies that we can view $P$ as a realization-by-realization improvement of $Q$, similar to the classic results on first-order stochastic dominance from the theory of risk. The axiom below, $F O S D$, says that if $P$ is a realization-by-realization improvement of $Q, P$ is preferred to $Q .{ }^{14}$

Axiom 8 (FOSD) For any $P, Q \in \Delta(\mathcal{F})$, if $P$ first-order stochastically dominates $Q$, then $P \succsim Q$

The next two axioms compare randomization with state-wise randomization. The first is related to a natural definition of second-order stochastic dominance in our setting. The discussion in Sections 1 and 2.1 shows that the extent to which randomization eliminates the effect of ambiguity depends on the individual's subjective timing belief, while the extent to which state-wise randomization eliminates the effect of ambiguity does not. Moreover, under

\footnotetext{
${ }^{14}$ One can show that FOSD is weaker than the relaxation of Strong Dominance in Saito (2011). In the context of social choice, the idea behind the monotonicity conditions in Gajdos and Maurin (2004) and Fleurbaey (2010) is similar to FOSD.
} 
the same mixture probability, state-wise randomization should be weakly more effective in eliminating the effect of ambiguity than randomization, regardless of the individual's subjective timing belief. Therefore, given any lottery, the individual should prefer to replace randomization with state-wise randomization whenever possible.

What choice behavior reveals that the individual prefers to replace randomization with state-wise randomization? First, we should have $\alpha f+_{s w}(1-\alpha) g \succsim \alpha f+(1-\alpha) g$ for any acts $f, g$ and $\alpha \in[0,1]$.

The comparison between $\alpha f+_{s w}(1-\alpha) g$ and $\alpha f+(1-\alpha) g$ closely resembles the comparison between $\delta_{\$(\alpha x+(1-\alpha) y)}$ and $\alpha \delta_{\$ x}+(1-\alpha) \delta_{\$ y}(x, y \in \mathbb{R})$ in expected utility theory. In particular, $\alpha f+_{s w}(1-\alpha) g$ and $\alpha f+(1-\alpha) g$ share the same expected utility (of prizes) fixing any state, similar to how $\delta_{\$(\alpha x+(1-\alpha) y)}$ and $\alpha \delta_{\$ x}+(1-\alpha) \delta_{\$ y}$ share the same expected amount of money. In expected utility theory, $\alpha \delta_{\$ x}+(1-\alpha) \delta_{\$ y}$ is a mean-preserving spread of $\delta_{\$(\alpha x+(1-\alpha) y)}$ (see Rothschild and Stiglitz (1970)). We define mean-preserving spreads in our setting following how they are defined in Rothschild and Stiglitz.

Definition 3 We say that $Q$ is a mean-preserving spread of $P$ if $P=\beta\left[\alpha f+_{s w}(1-\alpha) g\right]+$ $(1-\beta) R$ and $Q=\beta[\alpha f+(1-\alpha) g]+(1-\beta) R$ for some acts $f, g$, lottery $R$, and $\alpha, \beta \in[0,1]$.

When $Q$ is a mean-preserving spread of $P$ as defined above, some randomization in $Q$ involving the acts $f$ and $g$ is replaced with state-wise randomization in $P$. According to our previous discussion, the individual should prefer $P$ to $Q$, as stated in the axiom below.

Axiom 9 (SOSD) For any $P, Q \in \Delta(\mathcal{F})$, if $Q$ is a mean-preserving spread of $Q$, then $P \succsim Q$

The last axiom states that for constant acts, the individual remains indifferent between randomization and state-wise randomization, as in subjective expected utility theory. ${ }^{15}$ To understand this, recall that under $S O S D, P \succsim Q$ if $Q=\beta[\alpha f+(1-\alpha) g]+(1-\beta) R$ and

\footnotetext{
${ }^{15}$ A similar idea appears in an axiom called Indifference to Probability Mixture in Saito (2013).
} 
$P=\beta\left[\alpha f+_{s w}(1-\alpha) g\right]+(1-\beta) R$. Strict preference $P \succ Q$ occurs, however, only if $\alpha f+_{s w}(1-\alpha) g$ in $P$ is strictly more effective in eliminating the effect of ambiguity than $\alpha f+(1-\alpha) g$ in $Q$. Following Gilboa and Schmeidler (1989), we have assumed that when $g$ is a constant act, neither $\alpha f+_{s w}(1-\alpha) g$ nor $\alpha f+(1-\alpha) g$ eliminates the effect of ambiguity (see part (ii) of Preference for Randomization and part (ii) of Preference for State-wise Randomization). Thus, if $g$ is a constant act, $\alpha f+_{s w}(1-\alpha) g$ will not be strictly more effective than $\alpha f+(1-\alpha) g$ in eliminating the effect of ambiguity, and $P \succ Q$ should not occur. This observation is stated in our last axiom below.

We say that $P$ can be obtained by modifying $Q$ 's mixture timing of constant acts or $Q$ can be obtained by modifying $P$ 's mixture timing of constant acts if

$$
P=\beta\left[\alpha f+_{s w}(1-\alpha) p\right]+(1-\beta) R \text { and } Q=\beta[\alpha f+(1-\alpha) p]+(1-\beta) R
$$

for some act $f$, constant act $p$, lottery $R$, and $\alpha, \beta \in[0,1]$.

Axiom 10 (Indifference to Mixture Timing of Constant Acts) For any $P, Q \in \Delta(\mathcal{F})$, if $P$ can be obtained by modifying $Q$ 's mixture timing of constant acts, then $P \sim Q$.

This axiom rules out the possibility that, for example, the individual prefers early risk resolution or has different risk attitudes before and after the state is revealed. We can incorporate these possibilities in our analysis, but we leave them out to emphasize that our findings are not driven by these possibilities.

The following lemma complements Lemma 1 and shows that under some of our new axioms, Independence and State-wise Independence imply Strong Dominance. Together with Lemma 1, this means that it is impossible to only violate one axiom among Independence, State-wise Independence, and Strong Dominance. Any two of those three axioms imply the third. This finding will be useful later.

Lemma 2 Suppose $\succsim$ satisfies Weak Order, Continuity, FOSD, and Indifference to Mixture 
Timing of Constant Acts. Independence and State-wise Independence imply Strong Dominance.

Our main theorem will show that the axioms above are equivalent to the following representation of the individual's preference. Let $\mathcal{K}(\Delta(\mathcal{S}))$ be the collection of all nonempty closed subsets of $\Delta(\mathcal{S})$ endowed with the Hausdorff metric topology. Since $\Delta(\mathcal{S})$ is compact and Polish, $\mathcal{K}(\Delta(\mathcal{S}))$ is also compact and Polish (see Theorem 3.85 of Aliprantis and Border $(2006))$.

Definition 4 The individual's preference $\succsim$ has a double maxmin expected utility (DMEU) representation if there exist a continuous expected utility function $u: \Delta(\mathcal{X}) \rightarrow \mathbb{R}$ and $a$ compact collection $\mathcal{M}$ of compact subsets of $\Delta(\mathcal{S})$ such that $\succsim$ can be represented by

$$
W(P)=\min _{M \in \mathcal{M}} \int_{\mathcal{F}}\left(\min _{\mu \in M} \int_{\mathcal{S}} u(f) d \mu\right) d P
$$

If the individual's preference $\succsim$ has a representation described in the above definition, we say that $\succsim$ has a DMEU representation $(\mathcal{M}, u)$. Below is the main theorem.

Theorem 2 The individual's preference $\succsim$ has a DMEU representation if and only if it satisfies Weak Order, Continuity, Preference for Randomization, Preference for State-wise Randomization, FOSD, SOSD, and Indifference to Mixture Timing of Constant Acts.

The uniqueness of the representation will be studied in Section 3.2, and we provide some comparative static results in the Appendix.

In the DMEU representation, each $M \in \mathcal{M}$ is a set of priors called a scenario. The interpretation of the representation is that the individual who is conservative and focuses on the worst case believes that before she randomizes (trivially or not), a scenario $M \in \mathcal{M}$ unknown to her has occurred, and after the realization of her randomization, an unknown probability law from $M$ will be determined. Alternatively, the individual believes that before she randomizes, nature, which plays against her, has chosen a scenario from $\mathcal{M}$, and after she 
randomizes, nature will choose a probability law from the scenario based on the realization of her randomization.

Randomization may only partially eliminate the effect of ambiguity. It may help the individual hedge across scenarios, but not across probability laws within a scenario. Figure 5 is a simple example. If the individual chooses "Long" deterministically, the worst scenario will be $M_{B}$. If she chooses "Short" deterministically, the worst scenario will be $M_{G}$. Either way, her utility will be zero. However, if she randomizes between "Long" and "Short" with equal probability, the effect of ambiguity induced by $M_{G}$ and $M_{B}$ is eliminated: In scenarios $M_{G}$ and $M_{B}$, the individual receives the same prize with utility 0.5 . The worst scenario now is $M_{N}$, which contains two priors. Randomization cannot eliminate the effect of ambiguity induced by the priors in $M_{N}$, and the individual's utility will be 0.3 under $M_{N}$.

For any two acts $f$ and $g$, randomization between $f$ and $g$ may be strictly preferred only if $f$ and $g$ have different minimizing scenarios. With trivial randomization - that is, if the individual chooses an act $f$ with probability $1-(3)$ becomes

$$
W(f)=\min _{\mu \in \mathbb{M}} \int_{\mathcal{S}} u(f) d \mu
$$

in which $\mathbb{M}=\bigcup_{M \in \mathcal{M}} M$ pools all priors together.

\subsection{Sketch of the Proof}

To prove the sufficiency of the axioms, we begin by finding an expected utility representation for the individual's preference over constant acts, and use it to construct a utility representation $W(P)$ for her preference over lotteries.

Next, we define the expected utility core ${ }^{*}$ of $\succsim$ : For any lotteries $P, Q, P \succsim * Q$ if for any $R \in \Delta(\mathcal{F})$ and $\alpha \in(0,1], \alpha P+(1-\alpha) R \succsim \alpha Q+(1-\alpha) R$. Our notion of the expected utility core is similar to that of Cerreia-Vioglio (2009), Cerreia-Vioglio et al. (2015), and 
Cerreia-Vioglio, Maccheroni, and Marinacci (2017). ${ }^{16}$

We verify that ${ }^{*}$ satisfies the axioms in Dubra, Maccheroni, and Ok (2004) and then apply their main theorem to find a closed (and convex) set of continuous functions $\mathbb{V}$ such that $P \succsim^{*} Q$ if and only if

$$
\int_{\mathcal{F}} V\left(u\left(f_{1}\right), \ldots, u\left(f_{n}\right)\right) d P \geq \int_{\mathcal{F}} V\left(u\left(f_{1}\right), \ldots, u\left(f_{n}\right)\right) d Q
$$

for every $V \in \mathbb{V}$.

We show that if $P$ first-order stochastically dominates $Q$ or $Q$ is a mean-preserving spread of $P, P \succsim^{*} Q$. In addition, if $P$ can be obtained by modifying $Q$ 's mixture timing of constant acts, $P \sim^{*} Q$. According to the definition of $\succsim^{*}$, these observations imply that every $V \in \mathbb{V}$ satisfies properties similar to those in Gilboa and Schmeidler's (1989) Lemma 3.3, and therefore every $V \in \mathbb{V}$ has an MEU representation. Then, we use the fact that every $V$ has an MEU representation to establish that $\mathbb{V}$ is bounded and equicontinuous. According to the Arzelá-Ascoli theorem, $\mathbb{V}$ is compact.

Finally, we show that

$$
W(P)=\min _{V \in \mathbb{V}} \int_{\mathcal{F}} V\left(u\left(f_{1}\right), \ldots, u\left(f_{n}\right)\right) d P
$$

that is, the representation of the individual's preference $W(P)$ we initially constructed is equal to the minimum of $\int_{\mathcal{F}} V\left(u\left(f_{1}\right), \ldots, u\left(f_{n}\right)\right) d P \cdot{ }^{17}$ Note that for any lotteries $P$ and $Q$, $P{ }^{*} Q \Rightarrow P \succsim Q$, but the converse is not true in general. However, our axioms ensure that the converse holds under certain conditions, which is a key property of $\succsim^{*}$ for proving (4):

\footnotetext{
${ }^{16}$ Similar concepts have also appeared in earlier papers, including Nehring (2001) and Ghirardato, Maccheroni, and Marinacci (2004), which can be viewed as the dual version of the type of expected utility core that we use.

${ }^{17}$ The right-hand side of (4) is similar to the representation in Maccheroni (2002), but our choice domain, proof strategy, and research questions differ from his.
} 
For any lottery $P$ and constant act $p$,

$$
P \succsim p \Rightarrow P \succsim^{*} p
$$

A similar property of the expected utility core is also used in Cerreia-Vioglio et al. (2015).

\subsection{Uniqueness of the DMEU Representation}

Suppose the individual's preference is represented by the DMEU representation $W$ as in (3). In general, we can add redundant scenarios to $\mathcal{M}$ without changing $W$, and hence $\mathcal{M}$ will not be unique. For example, suppose $\mathcal{M}=\left\{\left\{\mu_{1}, \mu_{2}\right\}\right\}$ for some distinct $\mu_{1}, \mu_{2} \in \Delta(\mathcal{S})$. If we replace $\mathcal{M}$ with $\left\{\left\{\mu_{1}, \mu_{2}\right\},\left\{\mu_{1}\right\}\right\}$, because

$$
\int_{\mathcal{F}}\left(\min _{\mu \in\left\{\mu_{1}, \mu_{2}\right\}} \int_{\mathcal{S}} u(f) d \mu\right) d P \leq \int_{\mathcal{F}}\left(\min _{\mu \in\left\{\mu_{1}\right\}} \int_{\mathcal{S}} u(f) d \mu\right) d P
$$

the function $W$ will be unaffected. Once we remove the redundant scenarios from $\mathcal{M}$, however, $\mathcal{M}$ will essentially be unique.

Our proof of Theorem 2 in fact shows that the preference $\succsim$ satisfies the axioms if and only if it has a DMEU representation $(\mathcal{M}, u)$ such that $\mathcal{M}$ is a compact and convex collection of compact and convex subsets of $\Delta(\mathcal{S}) .{ }^{18}$ When a DMEU representation of $\succsim$ satisfies these additional convexity assumptions, we say that $\succsim$ has a DMEU* representation.

The result below shows that there is a unique DMEU* representation whose set of scenarios is minimal. The uniqueness of the expected utility function $u$ is standard: It is unique up to a positive affine transformation.

Theorem 3 Suppose the individual's preference $\succsim$ satisfies Weak Order, Continuity, Preference for Randomization, Preference for State-wise Randomization, FOSD, SOSD, and

\footnotetext{
${ }^{18}$ In the definition of the DMEU representation, we do not impose these additional convexity assumptions. This will keep our examples and discussion simple. For instance, the simple examples in Figures $3-5$ do not satisfy these convexity assumptions.
} 
Indifference to Mixture Timing of Constant Acts. Then, ¿ has a DMEU* representation $(\widehat{\mathcal{M}}, u)$ such that the following statements hold:

1. For any $D M E U^{*}$ representation $(\mathcal{M}, v)$ of $\succsim, \widehat{\mathcal{M}} \subset \mathcal{M}$ and $v=\alpha u+\beta$ for some $\alpha>0$ and $\beta \in \mathbb{R}$.

2. The expected utility core ${ }^{*}$ of $\succsim$ satisfies the following: For any $P, Q \in \Delta(\mathcal{F}), P \succsim^{*} Q$ if and only if

$$
\int_{\mathcal{F}}\left(\min _{\mu \in M} \int_{\mathcal{S}} u(f) d \mu\right) d P \geq \int_{\mathcal{F}}\left(\min _{\mu \in M} \int_{\mathcal{S}} u(f) d \mu\right) d Q
$$

for every $M \in \widehat{\mathcal{M}}$.

Moreover, $\widehat{\mathcal{M}}$ is unique.

Our notion of uniqueness is similar to that of Cerreia-Vioglio et al. (2015). In their continuous cautious expected utility representation, the minimal set of certainty equivalent functions is unique up to the closed convex hull.

\section{Special Cases of the DMEU}

We examine three special cases of the DMEU. In the first, it is as if the individual always believes that nature moves before she randomizes; in the second, it is as if she always believes that nature moves after she randomizes; and the third is Saito (2015). Epstein et al. (2007), Seo (2009), and Saito (2015) have studied representations similar to the first two special cases mentioned above.

To obtain the DMEU representation, we simultaneously relax Independence, State-wise Independence, and Strong Dominance. A natural question is what the representation of the individual's preference looks like if we only relax some of them. Lemmas 1 and 2 tell us that imposing any two of these three axioms is equivalent to imposing all of them, which leads 
to the SEU representation. Therefore, the remaining possibility is to impose exactly one of the three axioms on a preference with a DMEU representation.

First, we show that if Strong Dominance is imposed, it is as if the individual always believes that nature moves before she randomizes, which corresponds to Raiffa (1961).

Theorem 4 Suppose the individual's preference $\succsim$ has a DMEU representation. Then, there exists a continuous expected utility function $u: \Delta(\mathcal{X}) \rightarrow \mathbb{R}$ and a compact set $\mathbb{M} \subset \Delta(\mathcal{S})$ such that

$$
W^{e a}(P)=\min _{\mu \in \mathbb{M}} \int_{\mathcal{F}}\left(\int_{\mathcal{S}} u(f) d \mu\right) d P
$$

represents $\succsim$ if and only if $\succsim$ satisfies Strong Dominance.

We call (5) the Ex Ante $M E U$ representation. This representation is equivalent to the DMEU representation whose scenarios are all singletons, and the union of whose scenarios is $\mathbb{M}$. In the Ex Ante MEU representation, for each prior $\mu \in \mathbb{M}$, the individual evaluates a lottery $P$ using the standard SEU formula. Therefore, it is as if the individual believes that before she randomizes, a fixed but unknown probability law has already been determined.

To understand why the Ex Ante MEU representation satisfies Strong Dominance, recall that an important consequence of Strong Dominance is $P \sim f^{P}$, which is generally not true in the DMEU representation. However, in the Ex Ante MEU representation, $P \sim f^{P}$ always holds:

$$
\begin{aligned}
W^{e a}(P) & =\min _{\mu \in \mathbb{M}} \int_{\mathcal{F}}\left(\int_{\mathcal{S}} u(f) d \mu\right) d P \\
& =\min _{\mu \in \mathbb{M}} \int_{\mathcal{S}}\left(\int_{\mathcal{F}} u(f) d P\right) d \mu \\
& =\min _{\mu \in \mathbb{M}} \int_{\mathcal{S}} u\left(f^{P}\right) d \mu=W^{e a}\left(f^{P}\right) .
\end{aligned}
$$

Next, we turn to Independence. If Independence is imposed instead, we will obtain a representation in which it is as if the individual always believes that nature moves after she randomizes. 
Theorem 5 Suppose the individual's preference $\succsim$ has a DMEU representation. Then, there exists a continuous expected utility function $u: \Delta(\mathcal{X}) \rightarrow \mathbb{R}$ and a compact set $\mathbb{M} \subset \Delta(\mathcal{S})$ such that

$$
W^{e p}(P)=\int_{\mathcal{F}}\left(\min _{\mu \in \mathbb{M}} \int_{\mathcal{S}} u(f) d \mu\right) d P
$$

represents $\succsim$ if and only if $\succsim$ satisfies Independence.

We call (6) the Ex Post MEU representation. This representation is equivalent to the DMEU representation that has only one scenario, $\mathbb{M}$. In the Ex Post MEU representation, to evaluate a lottery $P$, for each act that is a realization of the lottery, the individual takes the worst prior from $\mathbb{M}$ to evaluate the act. Therefore, it is as if the individual believes that nature moves only after the realization of her randomization.

The reason the Ex Post MEU representation satisfies Independence is simple: $W^{e p}(P)$ is linear in $P$.

Finally, if we impose State-wise Independence on a preference with a DMEU representation, we will return to the SEU representation. To see this, recall that in a DMEU representation, the utility of an act (degenerate lottery) $f$ is $W(f)=\min _{\mu \in \mathbb{M}} \int_{\mathcal{S}} u(f) d \mu$, in which $\mathbb{M}=\bigcup_{M \in \mathcal{M}} M$ pools together all priors from all scenarios. This is the MEU representation of preferences over acts from Gilboa and Schmeidler (1989). If State-wise Independence holds, $W(f)$ must be linear. Then, there can only be one prior in $\mathbb{M}$, which means that the DMEU representation must be an SEU representation.

\subsection{Saito (2015) and the DMEU Representation}

Saito (2015) observes that in the urn experiment, there are two intuitive ways to evaluate a lottery. Using our language, the two ways correspond to the two extreme views of randomization - the view that nature moves completely before the individual randomizes and the view that nature moves completely after. Saito characterizes a representation in which a lottery $P$ is evaluated by a convex combination of the Ex Ante MEU function and 
the Ex Post MEU function; that is, the utility of $P$ is equal to

$$
W(P)=\delta W^{e a}(P)+(1-\delta) W^{e p}(P)
$$

for some $\delta \in[0,1]$ and some Ex Ante MEU function $W^{e a}$ and Ex Post MEU function $W^{e p}$ that share the same set of priors $\mathbb{M}$. The parameter $\delta$ measures how useful randomization is in the elimination of ambiguity's effect. Clearly, the main idea of this representation is closely related to that of our DMEU representation, and we show below that in fact, Saito's (2015) representation is a special case of the DMEU representation.

Saito's (2015) representation generalizes the Ex Ante MEU and the Ex Post MEU representations, but is not flexible enough to capture the following version of the service example. Suppose there are four service providers. Two are in town A, and the other two in town B. In town A, the convention is that service providers provide precise contracts, although no one reads them. In town $\mathrm{B}$, the convention is that no contracts will be provided. The quality of the services in town B, however, is "as good as" that of town A and the individual knows that. Specifically, there are four states. In state $s_{i j}$, town A's $i$ th provider's service and town B's $j$ th provider's service are identical with payoffs being 1 for the individual, and the payoffs of the other services are $0, i=1,2$ and $j=1,2$. Consider two choice problems. In the first, the individual will only consider town A's services due, for example, to weather conditions. In the second, she will only consider town B's. According to our discussion, in the first problem, randomization eliminates the effect of ambiguity. In the second, the effect of ambiguity is unaffected by randomization. In (7), this individual must use $\delta=1$ in the first problem and $\delta=0$ in the second. This is not allowed, since $\delta$ is fixed in Saito. The DMEU representation, however, allows the individual to hold a subjective timing belief that is consistent with this example.

In terms of Saito's (2015) axioms, because he studies preferences over sets of acts, it is not straightforward to see which of his axioms is violated by this example. Intuitively, 
however, the violated axiom should be Dominance. Denote the 50-50 randomization between choosing the two service providers in town A by $P$, and the $50-50$ randomization between choosing the two service providers in town B by $Q$. Now, imagine that nature moves before the individual randomizes in both choice problems. In that case, $P$ and $Q$ will be equally good. Similarly, imagine that nature moves after she randomizes in both choice problems. In that case, $P$ and $Q$ will again be equally good. Since Saito's Dominance compares lotteries based only on these two hypothetical extreme timing beliefs, Dominance should imply that the individual is indifferent between $P$ and $Q$. According to our discussion, however, the individual may strictly prefer $P$ to $Q$.

It is not obvious why the objective function (7) is a special case of the DMEU representation. To see this directly from the representation, given a representation as in (7), for each $\hat{\mu} \in \mathbb{M}$, let $M_{\hat{\mu}}:=\left\{\delta \hat{\mu}+(1-\delta) \mu^{\prime}: \mu^{\prime} \in \mathbb{M}\right\}$. Let $\mathcal{M}:=\left\{M_{\hat{\mu}}: \hat{\mu} \in \mathbb{M}\right\}$. Since $\mathbb{M}$ is compact, verifying that $\mathcal{M}$ is compact is straightforward. Now, consider the following DMEU representation:

$$
W(P)=\min _{M_{\hat{\mu}} \in \mathcal{M}} \int_{\mathcal{F}}\left(\min _{\mu \in M_{\hat{\mu}}} \int_{\mathcal{S}} u(f) d \mu\right) d P .
$$

We have

$$
\begin{aligned}
W(P) & =\min _{M_{\hat{\mu}} \in \mathcal{M}} \int_{\mathcal{F}}\left(\min _{\mu^{\prime} \in \mathbb{M}} \int_{\mathcal{S}} u(f) d\left(\delta \hat{\mu}+(1-\delta) \mu^{\prime}\right)\right) d P \\
& =\delta \min _{\hat{\mu} \in \mathbb{M}} \int_{\mathcal{F}} \int_{\mathcal{S}} u(f) d \hat{\mu} d P+(1-\delta) \int_{\mathcal{F}}\left(\min _{\mu^{\prime} \in \mathbb{M}} \int_{\mathcal{S}} u(f) d \mu^{\prime}\right) d P \\
& =\delta W^{e a}(P)+(1-\delta) W^{e p}(P) .
\end{aligned}
$$

Thus, the individual's objective function in Saito (2015) is a special case of the DMEU representation.

The observation above proves the following result. 
Theorem 6 Suppose the individual's preference $\succsim$ can be represented by

$$
W(P)=\delta \min _{\mu \in \mathbb{M}} \int_{\mathcal{F}}\left(\int_{\mathcal{S}} u(f) d \mu\right) d P+(1-\delta) \int_{\mathcal{F}}\left(\min _{\mu \in \mathbb{M}} \int_{\mathcal{S}} u(f) d \mu\right) d P
$$

for some compact and convex set $\mathbb{M} \subset \Delta(\mathcal{S})$ and $\delta \in[0,1]$. Then, $\succsim$ has a DMEU representation $(\mathcal{M}, u)$, in which $\mathcal{M}=\left\{M_{\hat{\mu}}: \hat{\mu} \in \mathbb{M}\right\}$ and $M_{\hat{\mu}}=\left\{\delta \hat{\mu}+(1-\delta) \mu^{\prime}: \mu^{\prime} \in \mathbb{M}\right\}$ for each $\hat{\mu} \in \mathbb{M}$.

This finding allows us to use the DMEU representation to offer a new interpretation of Saito's (2015) representation. Consider an individual who believes that the set of possible priors is a convex set $\mathbb{M}$. She believes that before she randomizes, nature has partially determined the probability law by choosing a subset of $\mathbb{M}$ or, equivalently, shrinking $\mathbb{M}$ to some subset of it. In particular, she believes that nature can shrink $\mathbb{M}$ by a fixed fraction, $1-\delta$, centered at any $\hat{\mu} \in \mathbb{M}$. Note that shrinking $\mathbb{M}$ by $1-\delta$ centered at $\hat{\mu} \in \mathbb{M}$ exactly generates the scenario $M_{\hat{\mu}}$ we construct above. Therefore, if an individual's preference can be represented by (7), it is as if she holds the above belief about how the probability law is determined.

\section{Discussion}

We have discussed several related papers at the end of the Introduction. We provide additional discussion in this section. In addition to Saito (2015), Seo (2009) and Saito (2011) are closely related to our paper. Seo characterizes the second-order subjective expected utility $(\mathrm{SOSEU})$ representation, in which the individual has a probability measure $m \in \Delta(\Delta(\mathcal{S}))$ over probability measures on the state space $(\mu$ 's from $\Delta(\mathcal{S})$ ), and she evaluates a lottery $P$ according to

$$
W(P)=\int_{\mathcal{F}}\left[\int_{\Delta(\mathcal{S})} v\left(\int_{\mathcal{S}} u(f) d \mu\right) d m(\mu)\right] d P .^{19}
$$

\footnotetext{
${ }^{19}$ This representation is similar to the smooth ambiguity representation introduced by Klibanoff, Marinacci, and Mukerji (2005).
} 
In terms of axioms, there are two main differences between the SOSEU representation and the DMEU representation. First, the former maintains Independence, while the latter relaxes it. Thus, in Seo, the individual always disagrees with Raiffa's argument that randomization renders ambiguity irrelevant and has no preference for randomization. Second, the DMEU representation satisfies Indifference to Mixture Timing of Constant Acts, while the SOSEU representation reduces to the SEU representation if a similar additional axiom is imposed (see Seo's Corollary 5.2). Hence, the only intersection between Seo's representation and ours is the SEU representation. Finally, as the function $v$ in Seo's representation becomes arbitrarily concave, the SOSEU representation will converge to the Ex Post MEU representation.

Noting that there are two popular ways to evaluate randomization under ambiguity, which correspond to what we call the Ex Ante MEU and the Ex Post MEU representations, Saito (2011) introduces a representation that combines them (see (7)). This representation later appears in Saito (2015), who, by analyzing preferences over sets of acts, characterizes the behavior of an individual who anticipates choosing randomly from a set of acts. Section 4.1 discusses some main differences between Saito's model and ours. In particular, since the DMEU representation nests Saito's representation as a special case, the latter must also satisfy $S O S D$. In other words, fixing any mixture probability, state-wise randomization must be more effective than randomization in eliminating the effect of ambiguity in Saito's representation.

The choice domain we use is from AA. By analyzing the individual's preference in the choice domain $\Delta(\mathcal{F})$, AA characterize the SEU representation. If their axiom, Reversal of Order in Compound Lotteries - or, in our version, Strong Dominance-holds, the individual does not distinguish between randomization and state-wise randomization. Then, the choice domain $\Delta(\mathcal{F})$ can be reduced to $\mathcal{F}$, in which case only state-wise randomization needs to be considered. Many papers on ambiguity have been built on this reduced version of AA's domain, either for the reason above or because randomization is not considered from the beginning; these include Gilboa and Schmeidler (1989); Schmeidler (1989); Ghirardato and 
Marinacci (2001); Maccheroni, Marinacci, and Rustichini (2006); Siniscalchi (2009); CerreiaVioglio, Maccheroni, Marinacci, and Montrucchio (2011); Lehrer and Teper (2011); and Frick et al. (2019). In contrast, Seo (2009), Saito (2011), and our paper adopt the original choice domain of AA without imposing Strong Dominance.

A large body of research has studied ambiguity and robustness (some maxmin principles) in various areas of economics. However, how mixed strategies should be introduced and evaluated has been an issue. For example, most papers that study monopoly sales problems have assumed that randomization can help individuals hedge (similar to the case in the Ex Ante MEU representation). ${ }^{20}$ On the other hand, most papers on contracts/auctions/mechanisms with ambiguity or robustness concerns have either ruled out randomization/mixed strategies or assumed that randomization does not help individuals hedge (similar to the case in the Ex Post MEU representation). ${ }^{21}$ Some of these papers point out that randomization should help individuals hedge, but this case will be more difficult to deal with. ${ }^{22}$ An exception is Bose and Renou (2014), who examine both extreme cases. Mixed strategies are ruled out in most papers on robustness or ambiguity in macroeconomics and finance. At least in some of these papers, it is natural to consider mixed strategies. ${ }^{23}$

\section{Conclusion}

How randomization/mixed strategies should be modeled in the presence of ambiguity or when some maxmin principle is used has been an unsettled issue in many research areas of economics. Two simple yet opposite ways to model randomization are well known. In the classic urn experiment of Ellsberg (1961), one corresponds to Raiffa (1961) — by which

\footnotetext{
${ }^{20}$ See, among others, Linhart and Radner (1989); Bergemann and Schlag (2008, 2011); Auster (2018); and Carrasco et al. (2018).

${ }^{21}$ See, among others, Bose, Ozdenoren, and Pape (2006); Bose and Daripa (2009); Bodoh-Creed (2012); Carroll (2015, 2017); Wolitzky (2016); de Castro, Liu, and Yannelis (2017a,b); Di Tillio et al. (2017); de Castro and Yannelis (2018); and Carroll and Segal (2019).

${ }^{22}$ According to our analysis, some of these papers (e.g., Di Tillio et al. (2017)) should have assumed that randomization helps individuals hedge due to their timing assumptions, but they do not.

${ }^{23}$ See, among others, Easley and O'Hara (2010); Epstein and Schneider (2010); Antić (2014); Easley et al. (2014); and Ilut, Kehrig, and Schneider (2018).
} 
randomization should render ambiguity irrelevant - and the other argues that the effect of ambiguity is unaffected by randomization.

We point out that the individual's belief about how the unknown probability law is determined is important in answering this question. If the individual believes that before she randomizes, the probability law - although unknown to her-has been determined, then the first way to model randomization is reasonable. If, however, she believes that the probability law is determined only after her randomization, then the second way is reasonable.

The subjective belief about how the unknown probability law is determined, however, is usually more complicated than these two extreme cases. For example, the probability that an investment is successful may depend on some long-term factor and some short-term factor. The individual may believe that before she makes any decision (possibly randomized), the unknown long-term factor has been determined, while the short-term factor will be determined much later. In this case, neither of these two ways to model randomization seems appropriate.

To address these issues, we adopt the classic framework of Anscombe and Aumann (1963) and relax their axioms. A new representation is derived. In the representation, the individual has a collection of sets of priors, $\mathcal{M}$. She behaves as if she believes that before she randomizes (trivially or not), nature has chosen an unknown scenario (a set of priors) $M \in \mathcal{M}$, and after the randomization, nature will choose an unknown prior from $M$. Thus, the individual's preference reveals her belief about how the unknown probability law is determined, and the representation allows randomization to partially eliminate the effect of ambiguity: Randomization may help the individual hedge across scenarios, but not across probability laws within a scenario. 


\section{References}

Agranov, M. and P. Ortoleva (2017). Stochastic Choice and Preferences for Randomization. Journal of Political Economy 125(1), 40-68.

Aliprantis, D. and K. Border (2006). Infinite Dimensional Analysis (3 ed.). Berlin Heidelberg: Springer-Verlag.

Anscombe, F. and R. Aumann (1963). A Definition of Subjective Probability. Annals of Mathematical Statistics 34(1), 199-205.

Antić, N. (2014). Contracting with Unknown Technologies. mimeo.

Auster, S. (2018). Robust Contracting Under Common Value Uncertainty. Theoretical Economics 13(1), 175-204.

Bade, S. (2015). Randomization Devices and the Elicitation of Ambiguity-Averse Preferences. Journal of Economic Theory 159(A), 221-235.

Baillon, A., Y. Halevy, and C. Li (2019). Experimental Elicitation of Ambiguity Attitude Using the Random Incentive System. mimeo.

Bergemann, D. and K. Schlag (2008). Pricing without Priors. Journal of the European Economic Association 6(2/3), 560-569.

Bergemann, D. and K. Schlag (2011). Robust Monopoly Pricing. Journal of Economic Theory $146(6), 2527-2543$.

Bodoh-Creed, A. (2012). Ambiguous Beliefs and Mechanism Design. Games and Economic Behavior $75(2), 518-537$. 
Bose, S. and A. Daripa (2009). A Dynamic Mechanism and Surplus Extraction Under Ambiguity. Journal of Economic Theory 144(5), 2084-2114.

Bose, S., E. Ozdenoren, and A. Pape (2006). Optimal Auctions with Ambiguity. Theoretical Economics 1(4), 411-438.

Bose, S. and L. Renou (2014). Mechanism Design with Ambiguous Communication Devices. Econometrica 82(5), 1853-1872.

Carrasco, V., V. Farinha Luz, N. Kos, M. Messner, P. Monteiro, and H. Moreira (2018). Optimal Selling Mechanisms Under Moment Conditions. Journal of Economic Theory 17r, $245-279$.

Carroll, G. (2015). Robustness and Linear Contracts. American Economic Review 105(2), $536-563$.

Carroll, G. (2017). Robustness and Separation in Multidimensional Screening. Econometrica $85(2), 453-488$.

Carroll, G. and I. Segal (2019). Robustly Optimal Auctions with Unknown Resale Opportunities. Review of Economic Studies 86(4), 1527-1555.

Cerreia-Vioglio, S. (2009). Maxmin Expected Utility on a Subjective State Space: Convex Preferences under Risk. mimeo.

Cerreia-Vioglio, S., D. Dillenberger, and P. Ortoleva (2015). Cautious Expected Utility and the Certainty Effect. Econometrica 83(2), 693-728.

Cerreia-Vioglio, S., D. Dillenberger, P. Ortoleva, and G. Riella (2019). Deliberately Stochastic. American Economic Review 109(7), 2425-2445.

Cerreia-Vioglio, S., F. Maccheroni, and M. Marinacci (2017). Stochastic Dominance Analysis without the Independence Axiom. Management Science 63(4), 1097-1109. 
Cerreia-Vioglio, S., F. Maccheroni, M. Marinacci, and L. Montrucchio (2011). Uncertainty Averse Preferences. Journal of Economic Theory 146(4), 1275-1330.

de Castro, L., Z. Liu, and N. Yannelis (2017a). Ambiguous Implementation: The Partition Model. Economic Theory 63(1), 233-261.

de Castro, L., Z. Liu, and N. Yannelis (2017b). Implementation Under Ambiguity. Games and Economic Behavior 101, 20-33.

de Castro, L. and N. Yannelis (2018). Uncertainty, Efficiency and Incentive Compatibility: Ambiguity Solves the Conflict Between Efficiency and Incentive Compatibility. Journal of Economic Theory 177, 678-707.

Di Tillio, A., N. Kos, and M. Messner (2017). The Design of Ambiguous Mechanisms. Review of Economic Studies 84(1), 237-276.

Dominiak, A. and W. Schnedler (2011). Attitudes Toward Uncertainty and Randomization: An Experimental Study. Economic Theory 48(2-3), 289-312.

Dubra, J., F. Maccheroni, and E. Ok (2004). Expected Utility Theory without the Completeness Axiom. Journal of Economic Theory 115(1), 118-133.

Dwenger, N., D. Kübler, and G. Weizsäcker (2018). Flipping a Coin: Evidence from Laboratory and Field. Journal of Public Economics 167, 240-250.

Easley, D. and M. O'Hara (2010). Microstructure and Ambiguity. Journal of Finance 65(5), $1817-1846$.

Easley, D., M. O'Hara, and L. Yang (2014). Opaque Trading, Disclosure, and Asset Prices: Implications for Hedge Fund Regulation. Review of Financial Studies 27(4), 1190-1237.

Ellsberg, D. (1961). Risk, Ambiguity, and the Savage Axioms. Quarterly Journal of Economics 75(4), 643-669. 
Epstein, L., M. Marinacci, and K. Seo (2007). Coarse Contingencies and Ambiguity. Theoretical Economics 2(4), 355-394.

Epstein, L. and M. Schneider (2010). Ambiguity and Asset Markets. Annual Review of Financial Economics 2, 315-346.

Fleurbaey, M. (2010). Assessing Risky Social Situations. Journal of Political Economy $118(4), 649-680$.

Frick, M., R. Iijima, and Y. Le Yaouanq (2019). Boolean Representations of Preferences under Ambiguity. mimeo.

Fudenberg, D., R. Iijima, and T. Strzalecki (2015). Stochastic Choice and Revealed Perturbed Utility. Econometrica 83(6), 2371-2409.

Gajdos, T. and E. Maurin (2004). Unequal Uncertainties and Uncertain Inequalities: An Axiomatic Approach. Journal of Economic Theory 116(1), 93-118.

Ghirardato, P., F. Maccheroni, and M. Marinacci (2004). Differentiating Ambiguity and Ambiguity Attitude. Journal of Economic Theory 118(2), 133-173.

Ghirardato, P. and M. Marinacci (2001). Risk, Ambiguity and the Separation of Utility and Beliefs. Mathematics of Operations Research 26(4), 864-890.

Gilboa, I. and D. Schmeidler (1989). Maxmin Expected Utility with Non-Unique Prior. Journal of Mathematical Economics 18(2), 141-153.

Hansen, L. and T. Sargent (2010). Wanting Robustness in Macroeconomics. In Handbook of Monetary Economics, Volume 3B, Chapter 20, pp. 1097-1157. North Holland.

Ilut, C., M. Kehrig, and M. Schneider (2018). Slow to Hire, Quick to Fire: Employment Dynamics with Asymmetric Responses to News. Journal of Political Economy 126(5), 2011-2071. 
Klibanoff, P., M. Marinacci, and S. Mukerji (2005). A Smooth Model of Decision Making Under Ambiguity. Econometrica 73(6), 1849-1892.

Kuzmics, C. (2017). Abraham Wald's Complete Class Theorem and Knightian Uncertainty. Games and Economic Behavior 104, 666-673.

Lehmann, E. (1955). Ordered Families of Distributions. Annals of Mathematical Statistics $26(3), 399-419$.

Lehrer, E. and R. Teper (2011). Justifiable Preferences. Journal of Economic Theory 146(2), $762-774$.

Linhart, P. and R. Radner (1989). Minimax-Regret Strategies for Bargaining over Several Variables. Journal of Economic Theory 48(1), 152-178.

Maccheroni, F. (2002). Maxmin under Risk. Economic Theory 19(4), 823-831.

Maccheroni, F., M. Marinacci, and A. Rustichini (2006). Ambiguity Aversion, Robustness, and the Variational Representation of Preferences. Econometrica 74(6), 1447-1498.

Machina, M. (1985). Stochastic Choice Functions Generated from Deterministic Preferences over Lotteries. Economic Journal 95, 575-594.

Machina, M. and D. Schmeidler (1992). A More Robust Definition of Subjective Probability. Econometrica 60(4), 745-780.

Nehring, K. (2001). Ambiguity in the Context of Probabilistic Beliefs. mimeo.

Oechssler, J., H. Rau, and A. Roomets (2019). Hedging, Ambiguity, and the Reversal of Order Axiom. Games and Economic Behavior 117, 380-387.

Raiffa, H. (1961). Risk, Ambiguity, and the Savage Axioms: Comment. Quarterly Journal of Economics 75(4). 
Rothschild, M. and J. Stiglitz (1970). Increasing Risk: I. A Definition. Journal of Economic Theory 2(3), 225-243.

Saito, K. (2011). Preference for Randomization and Ambiguity Aversion. mimeo.

Saito, K. (2013). Social Preferences under Risk: Equality of Opportunity versus Equality of Outcome. American Economic Review 103(7), 3084-3101.

Saito, K. (2015). Preferences for Flexibility and Randomization under Uncertainty. American Economic Review 105(3), 1246-1271.

Schmeidler, D. (1989). Subjective Probability and Expected Utility without Additivity. Econometrica 57(3), 571-587.

Seo, K. (2009). Ambiguity and Second-Order Belief. Econometrica 77(5), 1575-1605.

Siniscalchi, M. (2009). Vector Expected Utility and Attitudes toward Variation. Econometrica $77(3), 801-855$.

Wolitzky, A. (2016). Mechanism Design with Maxmin Agents: Theory and an Application to Bilateral Trade. Theoretical Economics 11(3), 971-1004. 


\section{A Appendix}

Proof of Lemma 1: The first statement can be verified using equation (1) and Weak Order directly. Next, suppose Independence and Strong Dominance hold. Take any acts $f, g, h$ and $\alpha \in(0,1)$ such that $f \succsim g$. By Independence, we know that $\alpha f+(1-\alpha) h \succsim$ $\alpha g+(1-\alpha) h$. Using the first statement of the lemma, $\alpha f+(1-\alpha) h \sim \alpha f{ }_{s w}(1-\alpha) h$ and $\alpha g+(1-\alpha) h \sim \alpha g+_{s w}(1-\alpha) h$. Therefore, $\alpha f+_{s w}(1-\alpha) h \succsim \alpha g+_{s w}(1-\alpha) h$. Showing that $\alpha f+_{s w}(1-\alpha) h \succsim \alpha g+_{s w}(1-\alpha) h$ implies $f \succsim g$ is similar. Next, suppose State-wise Independence and Strong Dominance hold. Take any lotteries $P, Q, R$ and $\alpha \in(0,1)$ such that $P \succsim Q$. Due to the first statement of the lemma, $f^{P} \succsim f^{Q}$. By State-wise Independence, $\alpha f^{P}+_{s w}(1-\alpha) f^{R} \succsim \alpha f^{Q}+_{s w}(1-\alpha) f^{R}$. Applying the first statement of the lemma again, we know that $\alpha f^{P}+{ }_{s w}(1-\alpha) f^{R} \sim \alpha P+(1-\alpha) R$ and $\alpha f^{Q}+{ }_{s w}(1-\alpha) f^{R} \sim \alpha Q+(1-\alpha) R$. Therefore, $\alpha P+(1-\alpha) R \succsim \alpha Q+(1-\alpha) R$. Showing that $\alpha P+(1-\alpha) R \succsim \alpha Q+(1-\alpha) R$ implies $P \succsim Q$ is similar.

Proof of Lemma 2: Consider two lotteries $P$ and $Q$ such that $f_{i}^{P} \succsim f_{i}^{Q}$ for each $i \in\{1, \ldots, n\}$. By FOSD, $f^{P} \succsim f^{Q}$. For each $f \in \mathcal{F}$, by Weak Order and Continuity, we can find a constant act $p^{f}$ such that $f \sim p^{f}$ (see Lemma 4 for more details). Let $\left\{P_{l}\right\}_{l=1}^{\infty}$ be a sequence of finite-support lotteries that converges to $P$. Take an arbitrary $P_{l}$. By applying Independence finitely many times, we know that the lottery $\bar{P}_{l}:=\sum_{f \in \operatorname{supp}\left(P_{l}\right)} P_{l}(f) \cdot p^{f} \in$ $\Delta(\Delta(\mathcal{X}))$ is indifferent to $P_{l}=\sum_{f \in \operatorname{supp}\left(P_{l}\right)} P_{l}(f) \cdot f$. Similarly, due to State-wise Independence, state-wise randomization $\sum_{f \in \operatorname{supp}\left(P_{l}\right)} P_{l}(f) \cdot p^{f}$ is indifferent to $\sum_{f \in \operatorname{supp}\left(P_{l}\right)} P_{l}(f) \cdot f=f^{P_{l}}$. Moreover, we can obtain $\sum_{f \in \operatorname{supp}\left(P_{l}\right)} P_{l}(f) \cdot p^{f}$ by modifying $\bar{P}_{l}$ 's mixture timing of constant acts finitely many times. Thus, Weak Order and Indifference to Mixture Timing of Constant Acts imply that $P_{l} \sim \bar{P}_{l} \sim f^{P_{l}}$. Since $f^{P_{l}}$ converges to $f^{P}$ according to (1), by Continuity, $P \sim f^{P}$. The same arguments apply to $Q$. Therefore, $P \succsim Q$. 
Proof of Theorem 2: We first prove the sufficiency of the axioms. Preference for State-wise Randomization implies that for any $\alpha \in(0,1)$ and $p, q, r \in \Delta(\mathcal{X}), p \succ q$ implies $\alpha p+_{s w}(1-\alpha) r \succ \alpha q+_{s w}(1-\alpha) r$. Since $\mathcal{X}$ is Polish, $\Delta(\mathcal{X})$ is Polish, and hence $\mathcal{F}$ is closed in $\Delta(\mathcal{F})$ and the set of constant acts is closed in $\Delta(\mathcal{F})$ (see Chapter 15 of Aliprantis and Border (2006)). Therefore, by Continuity, for any $p \in \Delta(\mathcal{X}),\{q \in \Delta(\mathcal{X}): q \succsim p\}$ and $\{q \in \Delta(\mathcal{X}): p \succsim q\}$ are closed. According to expected utility theory, there exists a continuous expected utility function $u: \Delta(\mathcal{X}) \rightarrow \mathbb{R}$ such that $p \succsim q$ if and only if $u(p) \geq u(q)$, in which the mixture operation is " + sw."

Similarly, when restricting $\succsim$ to $\Delta(\Delta(\mathcal{X})), \succsim$ also satisfies Independence.

Lemma 3 Every lottery in $\Delta(\Delta(\mathcal{X}))$ preserves Independence.

Proof. We show that for any $p, q \in \Delta(\mathcal{X})$ and $\alpha \in(0,1), \alpha p+(1-\alpha) q$ also preserves Independence. Take any $P, Q \in \Delta(\mathcal{F})$. Because $p$ preserves Independence, for any $\beta \in(0,1)$, $P \succsim Q$ if and only if $\beta P+(1-\beta) p \succsim \beta Q+(1-\beta) p$. Because $q$ preserves Independence, for any $\gamma \in(0,1), \beta P+(1-\beta) p \succsim \beta Q+(1-\beta) p$ if and only if

$$
\gamma(\beta P+(1-\beta) p)+(1-\gamma) q \succsim \gamma(\beta Q+(1-\beta) p)+(1-\gamma) q .
$$

In particular, let $\gamma=\frac{\alpha}{1-\beta+\alpha \beta}$, which implies that

$$
\frac{\gamma(1-\beta)}{1-\gamma}=\frac{\alpha}{1-\alpha}
$$

Clearly, $\gamma \in(\alpha, 1)$ and

$$
\beta \gamma=\frac{\alpha \beta}{1-\beta+\alpha \beta}
$$


can take any value in $(0,1)$ since $\alpha$ and $\beta$ are arbitrary numbers in $(0,1)$. Then, $(8)$ becomes

$$
\beta \gamma P+(1-\beta \gamma)(\alpha p+(1-\alpha) q) \succsim \beta \gamma Q+(1-\beta \gamma)(\alpha p+(1-\alpha) q)
$$

for any $\beta \gamma \in(0,1)$. Therefore, $\alpha p+(1-\alpha) q$ preserves Independence.

A standard induction argument implies that all lotteries in $\Delta(\Delta(\mathcal{X})$ ) (randomization over constant acts $\Delta(\mathcal{X})$ ) with finite support perserve Independence. Since $\mathcal{X}$ is Polish, $\Delta(\mathcal{X})$ is Polish, and therefore lotteries with finite support are dense in $\Delta(\Delta(\mathcal{X})$ ) (Theorem 15.10 of Aliprantis and Border (2006)). By Continuity, all lotteries in $\Delta(\Delta(\mathcal{X}))$ perserve Independence.

Since $\Delta(\Delta(\mathcal{X}))$ is a closed subset of $\Delta(\mathcal{F})$ (Corollary 15.6 of Aliprantis and Border $(2006)), \succsim$ on $\Delta(\Delta(\mathcal{X}))$ satisfies the standard continuity axiom. Thus, there exists a continuous expected utility function $u^{e a}: \Delta(\Delta(\mathcal{X})) \rightarrow \mathbb{R}$ such that for any $P, Q \in \Delta(\Delta(\mathcal{X}))$, $P \succsim Q$ if and only if $u^{e a}(P) \geq u^{e a}(Q)$, in which the mixture operation is " + ."

Because $\mathcal{X}$ is compact, $u$ and $u^{e a}$ are both continuous expected utility functions, and they represent the same preference on $\Delta(\mathcal{X})$, we must be able to find a best $x_{h}$ and a worst $x_{l}$ such that $u\left(\delta_{x_{h}}\right)=u^{e a}\left(\delta_{x_{h}}\right)=1$ and $u\left(\delta_{x_{l}}\right)=u^{e a}\left(\delta_{x_{l}}\right)=0$, and $u(\Delta(\mathcal{X}))=$ $u^{e a}(\Delta(\mathcal{X}))=[0,1] .{ }^{24}$ For any $p \in \Delta(\mathcal{X})$, we can find a unique $\alpha^{p} \in[0,1]$ such that $p \sim \alpha^{p} \delta_{x_{h}}+\left(1-\alpha^{p}\right) \delta_{x_{l}}$ (see Lemma 4 for more details). By Indifference to Mixture Timing of Constant Acts, $\alpha^{p} \delta_{x_{h}}+\left(1-\alpha^{p}\right) \delta_{x_{l}} \sim \alpha^{p} \delta_{x_{h}}+{ }_{s w}\left(1-\alpha^{p}\right) \delta_{x_{l}}$. Then, we have $u(p)=u^{e a}(p)$ any $p \in \Delta(\mathcal{X})$, which in turn implies that for any $P \in \Delta(\Delta(\mathcal{X})), u\left(f^{P}\right)=u^{e a}(P)$. Therefore, from here on, with an abuse of notation, we use $u$ to denote $u^{e a}$; that is, $u$ is a continuous expected utility representation of $\succsim$ restricted to $\Delta(\Delta(\mathcal{X}))$ under the mixture operation "+," and also of $\succsim$ restricted to $\Delta(\mathcal{X})$ under the mixture operation " ${ }_{s w}$." For each act $f$, let $\mathbf{u}(f)$ denote the $n$-tuple $\left(u\left(f_{1}\right), \ldots, u\left(f_{n}\right)\right) \in[0,1]^{n}$.

Each lottery $P \in \Delta(\mathcal{F})$ induces a Borel probability measure $m^{P}$ on $[0,1]^{n}$. Take any Borel set $\Lambda \subset[0,1]^{n}$. Recall that $\mathcal{F}$ is a closed subset of $\Delta(\mathcal{F})$. Since $u$ is continuous,

\footnotetext{
${ }^{24}$ Note that $u$ is linear on $\Delta(\mathcal{X})$, and $u^{e a}$ is linear on $\Delta(\Delta(\mathcal{X}))$.
} 
$\{f \in \mathcal{F}: \mathbf{u}(f) \in \Lambda\}$ is also Borel in $\Delta(\mathcal{F})$. Then, we define $m^{P}(\Lambda):=P(\{f \in \mathcal{F}: \mathbf{u}(f) \in \Lambda\})$ for each Borel set $\Lambda \subset[0,1]^{n}$. Verifying that $m^{P}$ is a probability measure is standard. This definition implies that for any $P, Q \in \Delta(\mathcal{F}), \alpha \in(0,1)$, and Borel set $\Lambda \subset[0,1]^{n}$,

$$
\begin{aligned}
m^{\alpha P+(1-\alpha) Q}(\Lambda) & =[\alpha P+(1-\alpha) Q](\{f \in \mathcal{F}: \mathbf{u}(f) \in \Lambda\}) \\
& =\alpha P(\{f \in \mathcal{F}: \mathbf{u}(f) \in \Lambda\})+(1-\alpha) Q(\{f \in \mathcal{F}: \mathbf{u}(f) \in \Lambda\}) \\
& =\alpha m^{P}(\Lambda)+(1-\alpha) m^{Q}(\Lambda) ;
\end{aligned}
$$

that is, $m^{\alpha P+(1-\alpha) Q}$ and $\alpha m^{P}+(1-\alpha) m^{Q}$ are the same measure.

Clearly, $\left\{m^{P}: P \in \Delta(\mathcal{F})\right\}$ is a subset of $\Delta\left([0,1]^{n}\right)$, the set of all Borel probability measures on $[0,1]^{n}$. The converse also holds. For each $\left(\alpha_{1}, \ldots, \alpha_{n}\right) \in[0,1]^{n}$, we can find an act $f^{\left(\alpha_{1}, \ldots, \alpha_{n}\right)}$ such that $f_{i}^{\left(\alpha_{1}, \ldots, \alpha_{n}\right)}=\alpha_{i} \delta_{x_{h}}+\left(1-\alpha_{i}\right) \delta_{x_{l}}{ }^{25}$ By definition, $\mathbf{u}\left(f^{\left(\alpha_{1}, \ldots, \alpha_{n}\right)}\right)=$ $\left(\alpha_{1}, \ldots, \alpha_{n}\right)$. Note that $F^{h l}:=\left\{f^{\left(\alpha_{1}, \ldots, \alpha_{n}\right)}:\left(\alpha_{1}, \ldots, \alpha_{n}\right) \in[0,1]^{n}\right\}$ is closed in $\mathcal{F}$ and thus in $\Delta(\mathcal{F})$. Take an arbitrary $m \in \Delta\left([0,1]^{n}\right)$. For any Borel measurable set $F \subset \mathcal{F}$, define $\Lambda^{F}:=\left\{\left(\alpha_{1}, \ldots, \alpha_{n}\right) \in[0,1]^{n}:\left(\alpha_{1}, \ldots, \alpha_{n}\right)=\mathbf{u}(f)\right.$ for some $\left.f \in F \cap F^{h l}\right\}$. Since $F \cap F^{h l}$ is Borel and $u$ is continuous, $\Lambda^{F}$ is Borel measurable. Let $P^{m}(F)=m\left(\Lambda^{F}\right)$. It is standard to verify that $P^{m} \in \Delta(\mathcal{F})$. Therefore, $\left\{m^{P}: P \in \Delta(\mathcal{F})\right\}=\Delta\left([0,1]^{n}\right)$.

For simplicity, for any $x \in \mathcal{X}$, we write $x$ instead of $\delta_{x}$. Then, $x$ may be treated as a consequence, a degenerate prize, a constant act, or a degenerate lottery, depending on the context. Next, we use $u$ to construct a representation of $\succsim$.

Lemma 4 There exists a continuous function $I: \Delta\left([0,1]^{n}\right) \rightarrow \mathbb{R}_{+}$such that for any $P, Q \in$ $\Delta(\mathcal{F})$, (a) $P \succsim Q$ if and only if $I\left(m^{P}\right) \geq I\left(m^{Q}\right)$; (b) $I\left(m^{P}\right)=u(P)$ if $P \in \Delta(\Delta(\mathcal{X})$ ); and (c) $I\left(\Delta\left([0,1]^{n}\right)\right)=[0,1]$.

Proof. Take any $P \in \Delta(\mathcal{F})$. By Continuity, $\left\{\alpha \in[0,1]: \alpha x_{h}+(1-\alpha) x_{l} \succsim P\right\}$ and $\left\{\alpha \in[0,1]: P \succsim \alpha x_{h}+(1-\alpha) x_{l}\right\}$ are closed in $[0,1]$. Neither is empty, because FOSD implies that $x_{h} \succsim P \succsim x_{l}$ and hence 1 belongs to the former set and 0 belongs to the latter.

\footnotetext{
${ }^{25}$ The mixture used here is the standard mixture operation from expected utility theory.
} 
Because the union of those two sets is a connected set $[0,1]$, we know that the two sets cannot have empty intersection. Therefore, for any $P$, we can find some $\alpha^{P}$ such that

$$
\alpha^{P} x_{h}+\left(1-\alpha^{P}\right) x_{l} \sim P .
$$

Since $\succsim$ is nontrivial, $x_{h} \succ x_{l}$ and for any $\alpha>\alpha^{\prime}, \alpha x_{h}+(1-\alpha) x_{l} \succ \alpha^{\prime} x_{h}+\left(1-\alpha^{\prime}\right) x_{l}$. Therefore, $\alpha^{P}$ is unique for each $P \in \Delta(\mathcal{F})$. Define a function $\tilde{I}: \Delta(\mathcal{F}) \rightarrow[0,1]$ such that

$$
\tilde{I}(P)=\alpha^{P}
$$

For constant acts $x_{h}$ and $x_{l}, \tilde{I}\left(x_{h}\right)=1=u\left(x_{h}\right)$ and $\tilde{I}\left(x_{l}\right)=0=u\left(x_{l}\right)$. It is standard to verify that $\tilde{I}$ represents $\succsim$.

Note that if $P \in \Delta(\Delta(\mathcal{X})), P \sim \alpha^{P} x_{h}+\left(1-\alpha^{P}\right) x_{l}$ implies that $u(P)=u\left(\alpha^{P} x_{h}+(1-\right.$ $\left.\left.\alpha^{P}\right) x_{l}\right)$. Therefore, $\tilde{I}(P)=u(P)$ for any $P \in \Delta(\Delta(\mathcal{X}))$.

For each $m \in \Delta\left([0,1]^{n}\right)$, we can find some $P \in \Delta(\mathcal{F})$ such that $m=m^{P}$. Define $I(m)=\tilde{I}(P)$ for each $m \in \Delta\left([0,1]^{n}\right)$. Then, we obtain a function $I: \Delta\left([0,1]^{n}\right) \rightarrow[0,1]$. Note that $I$ is well defined: For any $P, Q \in \Delta(\mathcal{F})$ such that $m^{P}=m^{Q}$, we must have $P \sim Q$ and hence $\tilde{I}(P)=\tilde{I}(Q)$. This is because when $m^{P}=m^{Q}$, according to our definition, $P$ firstorder stochastically dominates $Q$ and $Q$ first-order stochastically dominates $P$. Therefore, by $F O S D, P \sim Q$.

By Continuity, I is continuous. To see this, take any sequence of Borel probability measures on $[0,1]^{n},\left\{m_{l}\right\}_{l=1}^{\infty}$, that converges to $m \in \Delta\left([0,1]^{n}\right)$. The sequence of lotteries $\left\{P^{m_{l}}\right\}_{l=1}^{\infty}$ must converge to $P^{m}$. Based on how we define $\tilde{I}, \tilde{I}\left(P^{m_{l}}\right)$ must converge to $\tilde{I}\left(P^{m}\right)$.

Last, since $I\left(\Delta\left([0,1]^{n}\right)\right)$ is connected, $I\left(\Delta\left([0,1]^{n}\right)\right)=[0,1]$.

The function $I$ satisfies two additional useful properties.

Lemma 5 For any $P, Q \in \Delta(\mathcal{F})$ and $\alpha \in[0,1], I\left(\alpha m^{P}+(1-\alpha) m^{Q}\right) \geq \alpha I\left(m^{P}\right)+(1-$ $\alpha) I\left(m^{Q}\right)$. 
Proof. We know from Lemma 4 that $\alpha^{P} x_{h}+\left(1-\alpha^{P}\right) x_{l} \sim P$ and $\alpha^{Q} x_{h}+\left(1-\alpha^{Q}\right) x_{l} \sim Q$. By Lemma 3, we know that both $\alpha^{P} x_{h}+\left(1-\alpha^{P}\right) x_{l}$ and $\alpha^{Q} x_{h}+\left(1-\alpha^{Q}\right) x_{l}$ preserve Independence. Therefore,

$$
\begin{aligned}
& \alpha P+(1-\alpha)\left[\alpha^{Q} x_{h}+\left(1-\alpha^{Q}\right) x_{l}\right] \\
\sim & \alpha\left[\alpha^{P} x_{h}+\left(1-\alpha^{P}\right) x_{l}\right]+(1-\alpha)\left[\alpha^{Q} x_{h}+\left(1-\alpha^{Q}\right) x_{l}\right] \\
\sim & \alpha\left[\alpha^{P} x_{h}+\left(1-\alpha^{P}\right) x_{l}\right]+(1-\alpha) Q .
\end{aligned}
$$

By part (i) of Preference for Randomization,

$$
\begin{aligned}
& \frac{1}{2}\left(\alpha P+(1-\alpha)\left[\alpha^{Q} x_{h}+\left(1-\alpha^{Q}\right) x_{l}\right]\right)+\frac{1}{2}\left(\alpha\left[\alpha^{P} x_{h}+\left(1-\alpha^{P}\right) x_{l}\right]+(1-\alpha) Q\right) \\
\succsim & \alpha\left[\alpha^{P} x_{h}+\left(1-\alpha^{P}\right) x_{l}\right]+(1-\alpha) Q \\
\sim & \alpha\left[\alpha^{P} x_{h}+\left(1-\alpha^{P}\right) x_{l}\right]+(1-\alpha)\left[\alpha^{Q} x_{h}+\left(1-\alpha^{Q}\right) x_{l}\right] .
\end{aligned}
$$

Thus,

$$
\begin{aligned}
& \frac{1}{2}\left(\alpha P+(1-\alpha)\left[\alpha^{Q} x_{h}+\left(1-\alpha^{Q}\right) x_{l}\right]\right)+\frac{1}{2}\left(\alpha\left[\alpha^{P} x_{h}+\left(1-\alpha^{P}\right) x_{l}\right]+(1-\alpha) Q\right) \\
= & \frac{1}{2}(\alpha P+(1-\alpha) Q)+\frac{1}{2}\left(\alpha\left[\alpha^{P} x_{h}+\left(1-\alpha^{P}\right) x_{l}\right]+(1-\alpha)\left[\alpha^{Q} x_{h}+\left(1-\alpha^{Q}\right) x_{l}\right]\right) \\
\succsim & \alpha\left[\alpha^{P} x_{h}+\left(1-\alpha^{P}\right) x_{l}\right]+(1-\alpha)\left[\alpha^{Q} x_{h}+\left(1-\alpha^{Q}\right) x_{l}\right],
\end{aligned}
$$

which implies that $\alpha P+(1-\alpha) Q \succsim \alpha\left[\alpha^{P} x_{h}+\left(1-\alpha^{P}\right) x_{l}\right]+(1-\alpha)\left[\alpha^{Q} x_{h}+\left(1-\alpha^{Q}\right) x_{l}\right]$ since $\alpha\left[\alpha^{P} x_{h}+\left(1-\alpha^{P}\right) x_{l}\right]+(1-\alpha)\left[\alpha^{Q} x_{h}+\left(1-\alpha^{Q}\right) x_{l}\right]$ preserves Independence. Therefore, 
according to parts (a) and (b) of Lemma 4,

$$
\begin{aligned}
I\left(\alpha m^{P}+(1-\alpha) m^{Q}\right) & =I\left(m^{\alpha P+(1-\alpha) Q}\right) \\
& \geq I\left(m^{\alpha\left[\alpha^{P} x_{h}+\left(1-\alpha^{P}\right) x_{l}\right]+(1-\alpha)\left[\alpha^{Q} x_{h}+\left(1-\alpha^{Q}\right) x_{l}\right]}\right) \\
& =\alpha I\left(m^{\alpha^{P} x_{h}+\left(1-\alpha^{P}\right) x_{l}}\right)+(1-\alpha) I\left(m^{\alpha^{Q} x_{h}+\left(1-\alpha^{Q}\right) x_{l}}\right) \\
& =\alpha I\left(m^{P}\right)+(1-\alpha) I\left(m^{Q}\right) .
\end{aligned}
$$

Lemma 6 For any $P \in \Delta(\Delta(\mathcal{X})), Q \in \Delta(\mathcal{F})$, and $\alpha \in[0,1], I\left(\alpha m^{P}+(1-\alpha) m^{Q}\right)=$ $\alpha u(P)+(1-\alpha) I\left(m^{Q}\right)$.

Proof. According to Lemma 4, $Q \sim \alpha^{Q} x_{h}+\left(1-\alpha^{Q}\right) x_{l}$. Since $P$ preserves Independence (Lemma 3), $\alpha P+(1-\alpha) Q \sim \alpha P+(1-\alpha)\left[\alpha^{Q} x_{h}+\left(1-\alpha^{Q}\right) x_{l}\right]$. Then,

$$
\begin{aligned}
I\left(\alpha m^{P}+(1-\alpha) m^{Q}\right) & =I\left(m^{\alpha P+(1-\alpha) Q}\right) \\
& =I\left(m^{\alpha P+(1-\alpha)\left[\alpha^{Q} x_{h}+\left(1-\alpha^{Q}\right) x_{l}\right]}\right) \\
& =I\left(\alpha m^{P}+(1-\alpha) m^{\alpha^{Q} x_{h}+\left(1-\alpha^{Q}\right) x_{l}}\right) \\
& =\alpha u(P)+(1-\alpha) I\left(m^{\alpha^{Q} x_{h}+\left(1-\alpha^{Q}\right) x_{l}}\right) \\
& =\alpha u(P)+(1-\alpha) I\left(m^{Q}\right) .
\end{aligned}
$$

The fourth equality follows from the fact that both $\alpha^{Q} x_{h}+\left(1-\alpha^{Q}\right) x_{l}$ and $P$ are in $\Delta(\Delta(\mathcal{X}))$, and part (b) of Lemma 4.

Next, define $\succsim^{*}$ on $\Delta\left([0,1]^{n}\right)$ as follows: For any $P, Q \in \Delta(\mathcal{F})$,

$$
m^{P} \succsim^{*} m^{Q} \text { if } \alpha P+(1-\alpha) R \succsim \alpha Q+(1-\alpha) R
$$

for any $R \in \Delta(\mathcal{F})$ and $\alpha \in(0,1]$. Note that if there is another lottery $P^{\prime}$ such that $m^{P^{\prime}}=m^{P}$, by FOSD, $\alpha P+(1-\alpha) R \sim \alpha P^{\prime}+(1-\alpha) R$. Hence, $\succsim^{*}$ is well defined. We have the following 
observations.

Lemma 7 The following statements are true: (a) ${ }^{*}$ is reflexive and transitive, and for any $m \in \Delta\left([0,1]^{n}\right),\left\{m^{\prime} \in \Delta\left([0,1]^{n}\right): m^{\prime} \succsim^{*} m\right\}$ and $\left\{m^{\prime} \in \Delta\left([0,1]^{n}\right): m \succsim^{*} m^{\prime}\right\}$ are closed, (b) for any $P, Q, R \in \Delta(\mathcal{F})$ and $\alpha \in(0,1), m^{P} \succsim^{*} m^{Q}$ implies $\alpha m^{P}+(1-\alpha) m^{R} \succsim^{*}$ $\alpha m^{Q}+(1-\alpha) m^{R}$, and $(c)$ for any $P \in \Delta(\mathcal{F})$ and $Q \in \Delta(\Delta(\mathcal{X})), P \succsim Q$ implies $m^{P} \succsim^{*} m^{Q}$.

Proof. For part (a), reflexivity and transitivity of $\succsim^{*}$ are immediate. Since $\Delta\left([0,1]^{n}\right)$ is Polish, to verify the continuity property of ${ }^{*}$, it suffices to show that for any two sequences of lotteries $\left\{P_{l}\right\}$ and $\left\{Q_{l}\right\}$ such that $P_{l}$ converges to $P, Q_{l}$ converges to $Q$, and $m^{P_{l}} \succsim^{*} m^{Q_{l}}$ for any $l \in \mathbb{N}$, we have $m^{P} \succsim^{*} m^{Q}$. Fixing any $R \in \Delta(\mathcal{F})$ and $\alpha \in(0,1]$, since $\alpha P_{l}+(1-\alpha) R \succsim$ $\alpha Q_{l}+(1-\alpha) R$, by Continuity of $\succsim$, we have $\alpha P+(1-\alpha) R \succsim \alpha Q+(1-\alpha) R$.

For part (b), take any $R^{\prime} \in \Delta(\mathcal{F})$ and $\beta \in(0,1]$. We want to show that if $m^{P} \succsim^{*} m^{Q}$, we have

$$
\beta(\alpha P+(1-\alpha) R)+(1-\beta) R^{\prime} \succsim \beta(\alpha Q+(1-\alpha) R)+(1-\beta) R^{\prime}
$$

To see this, we only need to notice that $m^{P} \succsim^{*} m^{Q}$ and

$$
\beta(\alpha P+(1-\alpha) R)+(1-\beta) R^{\prime}=\alpha \beta P+(1-\alpha \beta)\left(\frac{\beta(1-\alpha)}{1-\alpha \beta} R+\frac{1-\beta}{1-\alpha \beta} R^{\prime}\right)
$$

and

$$
\beta(\alpha Q+(1-\alpha) R)+(1-\beta) R^{\prime}=\alpha \beta Q+(1-\alpha \beta)\left(\frac{\beta(1-\alpha)}{1-\alpha \beta} R+\frac{1-\beta}{1-\alpha \beta} R^{\prime}\right)
$$

To show $(\mathrm{c})$, take any $R \in \Delta(\mathcal{F})$. For any $\alpha \in(0,1]$, Lemmas 5 and 6 imply that

$$
\begin{aligned}
I\left(m^{\alpha P+(1-\alpha) R}\right) & =I\left(\alpha m^{P}+(1-\alpha) m^{R}\right) \\
& \geq \alpha I\left(m^{P}\right)+(1-\alpha) I\left(m^{R}\right) \\
& \geq \alpha I\left(m^{Q}\right)+(1-\alpha) I\left(m^{R}\right) \\
& =I\left(m^{\alpha Q+(1-\alpha) R}\right) .
\end{aligned}
$$


Consider the vector space of continuous functions from $[0,1]^{n}$ to $\mathbb{R}, C\left([0,1]^{n}\right)$, endowed with the sup norm. According to the above lemma and Dubra et al. (2004), there exist a closed and convex subset $\mathbb{V}$ of $C\left([0,1]^{n}\right)$ such that $m \succsim^{*} m^{\prime}$ if and only if

$$
\int_{[0,1]^{n}} V d m \geq \int_{[0,1]^{n}} V d m^{\prime} \text { for every } V \in \mathbb{V}
$$

Without loss of generality, assume that for every $V \in \mathbb{V}, V(0, \ldots, 0)=0$ and $V(1, \ldots, 1)=1$.

Next, we show that every $V$ in $\mathbb{V}$ satisfies a few properties. For any $\left(\alpha_{1}, \ldots, \alpha_{n}\right) \in[0,1]^{n}$, let $\delta_{\left(\alpha_{1}, \ldots, \alpha_{n}\right)}$ denote the Dirac measure on $\left(\alpha_{1}, \ldots, \alpha_{n}\right)$.

Lemma 8 Every $V$ in $\mathbb{V}$ is weakly increasing, concave, and satisfies $V\left(\lambda \alpha_{1}+(1-\lambda) \beta, \ldots, \lambda \alpha_{n}+\right.$ $(1-\lambda) \beta)=\lambda V\left(\alpha_{1}, \ldots, \alpha_{n}\right)+(1-\lambda) \beta$ for any $\left(\alpha_{1}, \ldots, \alpha_{n}\right) \in[0,1]^{n}$ and $\beta, \lambda \in[0,1]$.

Proof. For any $\left(\alpha_{1}, \ldots, \alpha_{n}\right),\left(\beta_{1}, \ldots, \beta_{n}\right) \in[0,1]^{n}$, we can find some $f$ and $g$ such that $\mathbf{u}(f)=\left(\alpha_{1}, \ldots, \alpha_{n}\right)$ and $\mathbf{u}(g)=\left(\beta_{1}, \ldots, \beta_{n}\right)$. SOSD implies that for any $R \in \Delta(\mathcal{F})$, $\lambda \in[0,1]$, and $\gamma \in(0,1]$,

$$
\gamma\left(\lambda f+_{s w}(1-\lambda) g\right)+(1-\gamma) R \succsim \gamma(\lambda f+(1-\lambda) g)+(1-\gamma) R .
$$

This means that $\lambda f+_{s w}(1-\lambda) g \succsim^{*} \lambda f+(1-\lambda) g$, and hence $\delta_{\left(\lambda \alpha_{1}+(1-\lambda) \beta_{1}, \ldots, \lambda \alpha_{n}+(1-\lambda) \beta_{n}\right)} \succsim^{*}$ $\lambda \delta_{\left(\alpha_{1}, \ldots, \alpha_{n}\right)}+(1-\lambda) \delta_{\left(\beta_{1}, \ldots, \beta_{n}\right)}$. Therefore, for any $V \in \mathbb{V}$,

$$
V\left(\lambda \alpha_{1}+(1-\lambda) \beta_{1}, \ldots, \lambda \alpha_{n}+(1-\lambda) \beta_{n}\right) \geq \lambda V\left(\alpha_{1}, \ldots, \alpha_{n}\right)+(1-\lambda) V\left(\beta_{1}, \ldots, \beta_{n}\right) .
$$

Following similar steps, FOSD implies that every $V \in \mathbb{V}$ is weakly increasing. Finally, suppose $p \in \Delta(\mathcal{X})$ satisfies $u(p)=\beta$. Indifference to Mixture Timing of Constant Acts implies that for any $R \in \Delta(\mathcal{F})$ and $\gamma \in(0,1]$,

$$
\gamma\left(\lambda f+_{s w}(1-\lambda) p\right)+(1-\gamma) R \sim \gamma(\lambda f+(1-\lambda) p)+(1-\gamma) R
$$


that is, $\delta_{\left(\lambda \alpha_{1}+(1-\lambda) \beta, \ldots, \lambda \alpha_{n}+(1-\lambda) \beta\right)} \sim^{*} \lambda \delta_{\left(\alpha_{1}, \ldots, \alpha_{n}\right)}+(1-\lambda) \delta_{(\beta, \ldots, \beta)}$. This means that for any $V \in \mathbb{V}$

$$
V\left(\lambda \alpha_{1}+(1-\lambda) \beta, \ldots, \lambda \alpha_{n}+(1-\lambda) \beta\right)=\lambda V\left(\alpha_{1}, \ldots, \alpha_{n}\right)+(1-\lambda) V(\beta, \ldots, \beta)
$$

We only need to show that $V(\beta, \ldots, \beta)=\beta$. According to our construction of $u, u(p)=\beta$ implies that $\beta x_{h}+(1-\beta) x_{l} \sim p$. By Lemma 3, we have $\beta x_{h}+(1-\beta) x_{l} \sim^{*} p$. Thus, for any $V \in \mathbb{V}$,

$$
\beta V(1, \ldots, 1)+(1-\beta) V(0, \ldots, 0)=\beta=V(\beta, \ldots, \beta) .
$$

The lemma above implies that every $V \in \mathbb{V}$ is positively homogeneous (let $\beta=0$ ) and $V\left([0,1]^{n}\right)=[0,1]$. Therefore, $\mathbb{V}$ is bounded. Below, we show that $\mathbb{V}$ is compact.

Lemma $9 \mathbb{V}$ is compact.

Proof. Since $[0,1]^{n}$ is Polish, $C\left([0,1]^{n}\right)$ is also Polish (see Chapter 3.19 of Aliprantis and Border (2006)). Therefore, to show that $\mathbb{V}$ is compact, we only need to show that it is sequentially compact. Since $[0,1]^{n}$ is compact and $\mathbb{V}$ is bounded, by the Arzelá-Ascoli theorem, if we can show that $\mathbb{V}$ is equicontinuous, we know that for any sequence in $\mathbb{V}$, there is a subsequence that converges in sup norm. Of course, the limit is in $\mathbb{V}$, because $C\left([0,1]^{n}\right)$ is complete, $\mathbb{V}$ is closed, and hence $\mathbb{V}$ is complete.

Suppose $\mathbb{V}$ is not equicontinuous. Then, there exists some $\left(\alpha_{1}, \ldots, \alpha_{n}\right) \in[0,1]^{n}$ such that for some $\varepsilon>0$, for any $\omega \in(0,1)$, we can find some $\left(\beta_{1}^{\omega}, \ldots, \beta_{n}^{\omega}\right)$ and $V_{\omega} \in \mathbb{V}$ such that

$$
\left|V_{\omega}\left(\beta_{1}^{\omega}, \ldots, \beta_{n}^{\omega}\right)-V_{\omega}\left(\alpha_{1}, \ldots, \alpha_{n}\right)\right|>\varepsilon
$$

in which $\left(\beta_{1}^{\omega}, \ldots, \beta_{n}^{\omega}\right)$ is within the $\omega$-neighborhood of $\left(\alpha_{1}, \ldots, \alpha_{n}\right)$. Take a sequence of $\left\{\left(\beta_{1}^{\omega_{j}}, \ldots, \beta_{n}^{\omega_{j}}\right)\right\}_{j}$ such that (11) holds and $\omega_{j}$ converges to zero as $j$ goes to infinity. 
Suppose $\alpha_{i}=0$ or 1 for some $i \in\{1, \ldots, n\}$. Because we have $V\left(\lambda \alpha_{1}+(1-\lambda) \gamma, \ldots, \lambda \alpha_{n}+\right.$ $(1-\lambda) \gamma)=\lambda V\left(\alpha_{1}, \ldots, \alpha_{n}\right)+(1-\lambda) \gamma$ for every $V \in \mathbb{V}$ and $\lambda, \gamma \in(0,1)$, we can multiply $\left(\alpha_{1}, \ldots, \alpha_{n}\right)$ and each $\left(\beta_{1}^{\omega_{j}}, \ldots, \beta_{n}^{\omega_{j}}\right)$ by $\lambda$ and add some $(\gamma, \ldots, \gamma)$ to them so that (i) $\lambda \alpha_{i}+$ $(1-\lambda) \gamma \in(0,1)$ for every $i \in\{1, \ldots, n\}$ and (ii) we have a new version of (11) with the right-hand side being $\lambda \varepsilon$. Therefore, without loss of generality, assume that $\alpha_{i} \in(0,1)$ for every $j \in\{1, \ldots, n\}$. Then, we can also assume without loss of generality that for any $j \in \mathbb{N}$, $\beta_{i}^{\omega_{j}}>0$ for any $i \in\{1, \ldots, n\}$.

Since $V(\gamma, \ldots, \gamma)=\gamma$ and $V$ is weakly increasing for any $V \in \mathbb{V}$, we know that $V_{\omega_{j}}\left(\alpha_{1}, \ldots, \alpha_{n}\right)>0$. Since every $V \in \mathbb{V}$ is bounded by $1,(11)$ implies that for each $j \in \mathbb{N}$,

$$
\frac{\left|V_{\omega_{j}}\left(\alpha_{1}, \ldots, \alpha_{n}\right)-V_{\omega_{j}}\left(\beta_{1}^{\omega_{j}}, \ldots, \beta_{n}^{\omega_{j}}\right)\right|}{V_{\omega_{j}}\left(\alpha_{1}, \ldots, \alpha_{n}\right)}=\left|\frac{V_{\omega_{j}}\left(\beta_{1}^{\omega_{j}}, \ldots, \beta_{n}^{\omega_{j}}\right)}{V_{\omega_{j}}\left(\alpha_{1}, \ldots, \alpha_{n}\right)}-1\right|>\varepsilon .
$$

However, because every $V \in \mathbb{V}$ is positively homogeneous and weakly increasing,

$$
\min _{i \in\{1, \ldots, n\}} \frac{\beta_{i}^{\omega_{j}}}{\alpha_{i}} \leq \frac{V\left(\beta_{1}^{\omega_{j}}, \ldots, \beta_{n}^{\omega_{j}}\right)}{V\left(\alpha_{1}, \ldots, \alpha_{n}\right)} \leq \max _{i \in\{1, \ldots, n\}} \frac{\beta_{i}^{\omega_{j}}}{\alpha_{i}}
$$

Therefore, as $j$ goes to infinity, $\frac{V\left(\beta_{1}^{\omega_{j}}, \ldots, \beta_{n}^{\omega_{j}}\right)}{V\left(\alpha_{1}, \ldots, \alpha_{n}\right)}$ must converge to 1 . We have a contradiction.

Note that for any $V \in \mathbb{V}$ and $P \in \Delta(\mathcal{F})$,

$$
\int_{\mathcal{F}} V(\mathbf{u}(f)) d P=\int_{[0,1]^{n}} V d m^{P}
$$

Next, we show that

$$
I\left(m^{P}\right)=\min _{V \in \mathbb{V}} \int_{[0,1]^{n}} V d m^{P} .
$$

If we can show this, according to the proof of Lemma 4 ,

$$
W(P)=\min _{V \in \mathbb{V}} \int_{\mathcal{F}} V(\mathbf{u}(f)) d P
$$

represents $\succsim$. 
Lemma 10 For any $P \in \Delta(\mathcal{F}), I\left(m^{P}\right)=\min _{V \in \mathbb{V}} \int_{[0,1]^{n}} V d m^{P}$.

Proof. Let $\alpha:=\min _{V \in \mathbb{V}} \int_{[0,1]^{n}} V d m^{P}$. First, we show that $I\left(m^{P}\right) \leq \alpha$. If $\alpha=1$, the inequality holds according to part (c) of Lemma 4. If $\alpha<1$, let $\beta$ be an arbitrary number such that $\alpha<\beta<1$. We can find some $p \in \Delta(\mathcal{X})$ such that $u(p)=\beta$ (see the proof of Lemma 4). Then, for some $V \in \mathbb{V}$,

$$
\int_{[0,1]^{n}} V d m^{P}<\beta=V(\beta, \ldots, \beta)
$$

that is, $m^{P} \Varangle^{*} \delta_{(\beta, \ldots, \beta)}=m^{p}$. By part (c) of Lemma 7, this implies that $p \succ P$. Therefore, $I\left(\delta_{(\beta, \ldots, \beta)}\right)=\beta>I\left(m^{P}\right)$. Let $\beta$ converge to $\alpha$. We have $I\left(m^{P}\right) \leq \alpha$.

Second, we show that $I\left(m^{P}\right) \geq \alpha$. We can find some $q \in \Delta(\mathcal{X})$ such that $u(q)=\alpha$. Then, according to the representation of $\succsim^{*}, m^{P} \succsim^{*} \delta_{(\alpha, \ldots, \alpha)}=m^{q}$. According to the definition of $\succsim^{*}, P \succsim q$; that is, $I\left(m^{P}\right) \geq u(q)=\alpha$.

Notice that each $V \in \mathbb{V}$ induces a preference over $\mathcal{F}$. Because $V$ is continuous, weakly increasing, concave, and satisfies $V\left(\lambda \alpha_{1}+(1-\lambda) \beta, \ldots, \lambda \alpha_{n}+(1-\lambda) \beta\right)=\lambda V\left(\alpha_{1}, \ldots, \alpha_{n}\right)+$ $(1-\lambda) \beta$, we can follow the steps in Gilboa and Schmeidler (1989) to show that $V(\mathbf{u}(f))=$ $\min _{\mu \in M} \int_{\mathcal{S}} u(f) d \mu$ for some unique compact and convex subset of $\Delta(\mathcal{S})$.

Lemma 11 For any $V \in \mathbb{V}$, there exists a unique compact and convex $M \subset \Delta(\mathcal{S})$ such that $V(\mathbf{u}(f))=\min _{\mu \in M} \int_{\mathcal{S}} u(f) d \mu$ for any $f \in \mathcal{F}$.

Proof. Denote the preference induced by $V$ on $\mathcal{F}$ by $\succsim^{V}$; that is, $f \succsim^{V} g$ if $V(\mathbf{u}(f)) \geq$ $V(\mathbf{u}(g))$. The only mixture operation considered for $\succsim^{V}$ on $\mathcal{F}$ is state-wise randomization, $+_{s w}$. We know that $\left(\mathcal{F},+_{s w}\right)$ is a mixture space. Clearly, $\succsim^{V}$ is complete and transitive, and for any act $f$, the sets $\left\{g \in \mathcal{F}: g \succsim^{V} f\right\}$ and $\left\{g \in \mathcal{F}: f \succsim^{V} g\right\}$ are closed because $V$ is continuous and, as shown previously, $\mathcal{F}$ is a closed subset of $\Delta(\mathcal{F})$. Concavity of $V$ and $V\left(\lambda \alpha_{1}+(1-\lambda) \beta, \ldots, \lambda \alpha_{n}+(1-\lambda) \beta\right)=\lambda V\left(\alpha_{1}, \ldots, \alpha_{n}\right)+(1-\lambda) \beta$ imply that constant acts preserve State-wise Independence, and that for any $f, g$ and $\alpha \in(0,1), f \succsim^{V} g$ implies 
$\alpha f+_{s w}(1-\alpha) g \succsim^{V} g$. Lastly, FOSD implies that if for any $i \in\{1, \ldots, n\}, f_{i} \succsim^{V} g_{i}$, then $f \succsim^{V} g$. Therefore, we can apply the results of Gilboa and Schmeidler (1989) to show that there exists a unique compact and convex set $M_{V}$ of probability measures over $\mathcal{S}$ such that

$$
V(\mathbf{u}(f))=\min _{\mu \in M_{V}} \int_{\mathcal{S}} u(f) d \mu \text { for any } f \in \mathcal{F} .
$$

The compactness of $M_{V}$ follows from the fact that $M_{V}$ is a closed subset of the compact set $\Delta(\mathcal{S})$

The proof of Lemma 11 in fact implies a stronger result: There is a bijection $\phi$ (defined by (14)) from the collection of nonempty compact and convex subsets of $\Delta(\mathcal{S})$ to the set of functions in $C\left([0,1]^{n}\right)$ that satisfy properties described in Lemma 8. Let $\mathcal{M}=\left\{\phi^{-1}(V)\right.$ : $V \in \mathbb{V}\}$. The next lemma is not needed to prove Theorem 2, but will be useful for Theorem 3.

Lemma $12 \mathcal{M}$ is convex.

Proof. Take any $\lambda \in[0,1]$ and $M_{1}, M_{2} \in \mathcal{M}$. Let $V_{j}=\phi\left(M_{j}\right), j=1,2$. The following equality holds:

$$
\left(\lambda V_{1}+(1-\lambda) V_{2}\right)(\mathbf{u}(f))=\min _{\mu \in \lambda M_{1}+(1-\lambda) M_{2}} \int_{\mathcal{S}} u(f) d \mu
$$

for any $f \in \mathcal{F}$, in which $\lambda M_{1}+(1-\lambda) M_{2}=\left\{\lambda \mu_{1}+(1-\lambda) \mu_{2}: \mu_{1} \in M_{1}\right.$ and $\left.\mu_{2} \in M_{2}\right\}$. We only need to prove that $\lambda M_{1}+(1-\lambda) M_{2}$ is convex and compact.

Since $M_{1}$ and $M_{2}$ are convex, proving that $\lambda M_{1}+(1-\lambda) M_{2}$ is convex is straightforward. To prove that it is compact, take an arbitrary sequence in $\lambda M_{1}+(1-\lambda) M_{2}$, $\left\{\lambda \mu_{1}^{k}+(1-\lambda) \mu_{2}^{k}\right\}_{k=1}^{\infty}$. Note that $M_{1}$ and $M_{2}$ are compact. Since $\left\{\mu_{1}^{k}\right\}_{k=1}^{\infty}$ has a convergent subsequence, without loss of generality, let $\left\{\lambda \mu_{1}^{k}+(1-\lambda) \mu_{2}^{k}\right\}_{k=1}^{\infty}$ be a sequence such that $\left\{\mu_{1}^{k}\right\}_{k=1}^{\infty}$ converges. Then, because $\left\{\mu_{2}^{k}\right\}_{k=1}^{\infty}$ has a convergent subsequence $\left\{\mu_{2}^{k_{l}}\right\}_{l=1}^{\infty}$, the subsequence $\left\{\lambda \mu_{1}^{k_{l}}+(1-\lambda) \mu_{2}^{k_{l}}\right\}_{l=1}^{\infty}$ must converge.

Since $\lambda V_{1}+(1-\lambda) V_{2} \in \mathbb{V}, \lambda M_{1}+(1-\lambda) M_{2}=\phi^{-1}\left(\lambda V_{1}+(1-\lambda) V_{2}\right) \in \mathcal{M}$. 
Because $\mathcal{K}(\Delta(\mathcal{S}))$ is compact (Theorem 3.85 of Aliprantis and Border (2006)), if we can show that $\mathcal{M}$ is closed, $\mathcal{M}$ is compact. This is implied by the following lemma.

Lemma $13 \mathcal{M}$ is closed.

Proof. Take an arbitrary sequence $\left\{M_{j}\right\}_{j=1}^{\infty}$ in $\mathcal{M}$ that converges to some $M \in \mathcal{K}(\Delta(\mathcal{S}))$. Since $M \in \mathcal{K}(\Delta(\mathcal{S})), M$ is a closed subset of the compact set $\Delta(\mathcal{S})$ and hence $M$ is compact. Verifying that $M$ is convex is standard.

Let $V_{j}=\phi\left(M_{j}\right) \in \mathbb{V}$ for each $j \in \mathbb{N}$. Since $\mathbb{V}$ is compact, we know that $\left\{V_{j}\right\}_{j=1}^{\infty}$ has a convergent subsequence $\left\{V_{j_{k}}\right\}_{k=1}^{\infty}$ whose limit $V$ is in $\mathbb{V}$.

By definition, for any $f \in \mathcal{F}$,

$$
V(\mathbf{u}(f))=\min _{\mu \in \phi^{-1}(V)} \int_{\mathcal{S}} u(f) d \mu
$$

We need to prove that $M=\phi^{-1}(V) \in \mathcal{M}$. If this is not true, since $M$ is compact and convex, according to the uniqueness of the MEU representation, there must be some $g \in \mathcal{F}$ such that

$$
\left|V(\mathbf{u}(g))-\min _{\mu \in M} \int_{\mathcal{S}} u(g) d \mu\right|=\varepsilon>0 .
$$

Because $V_{j_{k}}(\mathbf{u}(g))=\min _{\mu \in M_{j_{k}}} \int_{\mathcal{S}} u(g) d \mu$ for each $k, V_{j_{k}}(\mathbf{u}(g))$ converges to $V(\mathbf{u}(g))$ as $k$ goes to infinity, and $u$ is a continuous function defined on a compact set, even when $k$ is large, there must be some $\mu \in M$ that is sufficiently distant from all priors in $M_{j_{k}}$ (in the case of $\left.\min _{\mu \in M} \int_{\mathcal{S}} u(g) d \mu<V(\mathbf{u}(g))\right)$, or some $\mu_{j_{k}} \in M_{j_{k}}$ that is sufficiently distant from all priors in $M$ (in the case of $\min _{\mu \in M} \int_{\mathcal{S}} u(g) d \mu>V(\mathbf{u}(g))$ ). Thus, $\left\{M_{j_{k}}\right\}_{k=1}^{\infty}$ does not converge to $M$. We have a contradiction.

To summarize, we have shown that if $\succsim$ satisfies the axioms in Theorem 2 , $\succsim$ has a DMEU representation $(\mathcal{M}, u)$ in which $\mathcal{M}$ is a compact and convex collection of compact and convex subsets of $\Delta(\mathcal{S})$. This will be called a DMEU* representation in Theorem 3.

Proving the necessity of the axioms is standard. We only verify FOSD and SOSD below. 
Suppose

$$
W(P)=\min _{M \in \mathcal{M}} \int_{\mathcal{F}}\left(\min _{\mu \in M} \int_{\mathcal{S}} u(f) d \mu\right) d P
$$

represents $\succsim$ such that $\mathbb{V}=\left\{\min _{\mu \in M} \int_{\mathcal{S}} u(f) d \mu: M \in \mathcal{M}\right\}$ is compact.

We verify FOSD for a more general representation described in (13), in which $\mathbb{V}$ is compact and every $V \in \mathbb{V}$ is continuous and weakly increasing. This representation nests the DMEU representation as a special case. Thus, if we can show that every $\succsim$ that has such a representation satisfies FOSD, then whenever $\succsim$ has a DMEU representation, FOSD must also hold.

Suppose $P$ first-order stochastically dominates $Q$. For each $V \in \mathbb{V}, \int V(\mathbf{u}(f)) d P \geq$ $\int V(\mathbf{u}(f)) d Q$ because $V$ is weakly increasing (see Lehmann (1955)). Therefore,

$$
\min _{V \in \mathbb{V}} \int V(\mathbf{u}(f)) d P=\int V^{P}(\mathbf{u}(f)) d P \geq \int V^{P}(\mathbf{u}(f)) d Q \geq \min _{V \in \mathbb{V}} \int V(\mathbf{u}(f)) d Q
$$

which means that FOSD holds.

Next, let $Q$ be a mean-preserving spread of $P$; that is, $P=\beta\left[\alpha f+_{s w}(1-\alpha) g\right]+(1-\beta) R$ and $Q=\beta[\alpha f+(1-\alpha) g]+(1-\beta) R$ for some $\beta \in[0,1]$ and $R \in \Delta(\mathcal{F})$. We need to verify that $W(P) \geq W(Q)$. We have

$$
\begin{aligned}
W(P) & =\min _{M \in \mathcal{M}}\left\{\beta\left(\min _{\mu \in M} \int_{\mathcal{S}} u((\alpha f+s w(1-\alpha) g)) d \mu\right)+(1-\beta) \int_{\mathcal{F}}\left(\min _{\mu \in M} \int_{\mathcal{S}} u(h) d \mu\right) d R\right\} \\
& =\min _{M \in \mathcal{M}}\left\{\beta\left(\min _{\mu \in M} \int_{\mathcal{S}}[\alpha u(f)+(1-\alpha) u(g)] d \mu\right)+(1-\beta) \int_{\mathcal{F}}\left(\min _{\mu \in M} \int_{\mathcal{S}} u(h) d \mu\right) d R\right\},
\end{aligned}
$$

and

$$
W(Q)=\min _{M \in \mathcal{M}}\left\{\begin{array}{c}
\alpha \beta\left(\min _{\mu \in M} \int_{\mathcal{S}} u(f) d \mu\right)+(1-\alpha) \beta\left(\min _{\mu \in M} \int_{\mathcal{S}} u(g) d \mu\right) \\
+(1-\beta) \int_{\mathcal{F}}\left(\min _{\mu \in M} \int_{\mathcal{S}} u(h) d \mu\right) d R
\end{array}\right\} .
$$


Notice that for each $M \in \mathcal{M}$,

$$
\min _{\mu \in M} \int_{\mathcal{S}}[\alpha u(f)+(1-\alpha) u(g)] d \mu \geq \alpha\left(\min _{\mu \in M} \int_{\mathcal{S}} u(f) d \mu\right)+(1-\alpha)\left(\min _{\mu \in M} \int_{\mathcal{S}} u(g) d \mu\right)
$$

Therefore, $W(P) \geq W(Q)$.

Proof of Theorem 3: The proof of Theorem 2 shows that we can find a continuous expected utility function $u: \Delta(\mathcal{X}) \rightarrow \mathbb{R}$ and a compact and convex set $\widehat{\mathbb{V}} \subset C\left([0,1]^{n}\right)$ such that (i) for any $m, m^{\prime} \in \Delta\left([0,1]^{n}\right), m \succsim^{*} m^{\prime}$ if and only if

$$
\int_{[0,1]^{n}} V d m \geq \int_{[0,1]^{n}} V d m^{\prime} \text { for every } V \in \widehat{\mathbb{V}}
$$

(ii) for each $V \in \widehat{\mathbb{V}}$, there exists a unique compact and convex set $\phi^{-1}(V) \subset \Delta(\mathcal{S})$ such that $V(\mathbf{u}(f))=\min _{\mu \in \phi^{-1}(V)} \int_{\mathcal{S}} u(f) d \mu$; and (iii) $(\widehat{\mathcal{M}}, u)$ is a DMEU* representation of $\succsim$ in which $\widehat{\mathcal{M}}=\left\{\phi^{-1}(V): V \in \widehat{\mathbb{V}}\right\}$. The function $\phi$ is the bijection defined in the proof of Theorem 2 .

Note that ${ }^{*}$ defined in (10) is different from the expected utility core we define in Section 3.1 , because $\succsim^{*}$ is a binary relation on $\Delta\left([0,1]^{n}\right)$ and the expected utility core is a binary relation on $\Delta(\mathcal{F})$. However, with an abuse of notation, letting ${ }^{*}$ also denote the expected utility core of $\succsim$, we immediately have $P \succsim^{*} Q \Longleftrightarrow m^{P} \succsim^{*} m^{Q}$ for any $P, Q \in \Delta(\mathcal{F})$, according to the definition of the expected utility core.

We know that for any $V \in \widehat{\mathbb{V}}$ and $P \in \Delta(\mathcal{F}), \int_{\mathcal{F}} V(\mathbf{u}(f)) d P=\int_{[0,1]^{n}} V d m^{P}$. Therefore, for any $P, Q \in \Delta(\mathcal{F}), P \succsim^{*} Q$ if and only if for every $V \in \widehat{\mathbb{V}}$,

$$
\begin{aligned}
\int_{\mathcal{F}} V(\mathbf{u}(f)) d P & \geq \int_{\mathcal{F}} V(\mathbf{u}(f)) d Q \\
\int_{\mathcal{F}}\left(\min _{\mu \in M_{V}} \int_{\mathcal{S}} u(f) d \mu\right) d P & \geq \int_{\mathcal{F}}\left(\min _{\mu \in M_{V}} \int_{\mathcal{S}} u(f) d \mu\right) d Q
\end{aligned}
$$


which means that the DMEU* representation $(\widehat{\mathcal{M}}, u)$ of $\succsim$ satisfies part 2 of the theorem.

Suppose there is another DMEU* representation $(\mathcal{M}, v)$ of $\succsim$. Since both $u$ and $v$ are expected utility representations of $\succsim$ on $\Delta(\mathcal{X})$, there exist $\alpha>0$ and $\beta \in R$ such that $v=\alpha u+\beta$. Because

$$
\begin{aligned}
W_{\mathcal{M}, v}(P) & =\min _{M \in \mathcal{M}} \int_{\mathcal{F}}\left(\min _{\mu \in M} \int_{\mathcal{S}} v(f) d \mu\right) d P \\
& =\alpha \min _{M \in \mathcal{M}} \int_{\mathcal{F}}\left(\min _{\mu \in M} \int_{\mathcal{S}} u(f) d \mu\right) d P+\beta \\
& =\alpha W_{\mathcal{M}, u}(P)+\beta,
\end{aligned}
$$

$(\mathcal{M}, v)$ is a $\mathrm{DMEU}^{*}$ representation of $\succsim$ if and only if $(\mathcal{M}, u)$ is a $\mathrm{DMEU}^{*}$ representation of $\succsim$. Thus, without loss of generality, let $v=u$. Let

$$
\mathbb{V}=\left\{\phi(M) \in C\left([0,1]^{n}\right): M \in \mathcal{M}\right\}
$$

Using arguments similar to the proof of Lemmas 12 and 13 , we can verify that $\mathbb{V}$ is compact and convex.

We first prove that $\widehat{\mathcal{M}}$ is unique. Suppose $(\mathcal{M}, u)$ also satisfies part 2 of the theorem. Then, for any $P, Q \in \Delta(\mathcal{F})$, the following three statements are equivalent: (i) $P \succsim^{*} Q$; (ii) for every $V \in \widehat{\mathbb{V}}, \int_{\mathcal{F}} V(\mathbf{u}(f)) d P \geq \int_{\mathcal{F}} V(\mathbf{u}(f)) d Q$; and (iii) for every $V \in \mathbb{V}, \int_{\mathcal{F}} V(\mathbf{u}(f)) d P \geq$ $\int_{\mathcal{F}} V(\mathbf{u}(f)) d Q$. According to the uniqueness result from Dubra et al. (2004), we know that

$$
\operatorname{cl}\left(\operatorname{cone}(\widehat{\mathbb{V}})+\left\{\gamma \mathbf{1}_{[0,1]^{n}}\right\}_{\gamma \in \mathbb{R}}\right)=\operatorname{cl}\left(\operatorname{cone}(\mathbb{V})+\left\{\gamma \mathbf{1}_{[0,1]^{n}}\right\}_{\gamma \in \mathbb{R}}\right)
$$

Note that $\mathbb{V}^{\prime}:=\operatorname{cl}\left(\operatorname{cone}(\mathbb{V})+\left\{\gamma \mathbf{1}_{[0,1]^{n}}\right\}_{\gamma \in \mathbb{R}}\right) \cap\left\{V \in C\left([0,1]^{n}\right): V(\lambda, \ldots, \lambda)=\lambda\right.$ for any $\lambda \in[0,1]\}=\overline{\mathrm{co}}(\mathbb{V})=\mathbb{V}$. To show this, since $\mathbb{V} \subset \mathbb{V}^{\prime}$, we only need to show that $V \in \mathbb{V}^{\prime} \Rightarrow V \in \mathbb{V}$. Suppose $V$ is the limit of a sequence $\left\{\theta_{k} V_{k}+\gamma_{k} \mathbf{1}_{[0,1]^{n}}\right\}_{k=1}^{\infty}$, in which $\theta_{k} \geq 0, \gamma_{k} \in \mathbb{R}$, and $V_{k} \in \operatorname{co}(\mathbb{V})$ for each $k \in \mathbb{N}$. Because $V \in \mathbb{V}^{\prime}$, it satisfies $V(\lambda, \ldots, \lambda)=\lambda$ for any $\lambda \in[0,1]$. Then, $V(0, \ldots, 0)=0$ implies that $\gamma_{k}$ must converge to 0 . This together 
with the fact that $V(1, \ldots, 1)=1$ implies that $\theta_{k}$ must converge to 1 . Therefore, it must be true that $V \in \overline{\mathrm{co}}(\mathbb{V})$. Since $\mathbb{V}$ is compact and convex, $\overline{\mathrm{co}}(\mathbb{V})=\mathbb{V}$. The same arguments hold for $\widehat{\mathbb{V}}$. Then, (15) implies that $\widehat{\mathbb{V}}=\mathbb{V}$. Since $\phi$ is a bijection, $\widehat{\mathcal{M}}=\mathcal{M}$.

Next, without assuming that the DMEU* representation $(\mathcal{M}, u)$ of $\succsim$ satisfies part 2 of the theorem, we show that $\widehat{\mathcal{M}} \subset \mathcal{M}$. First, define $\succsim^{\prime}$ on $\Delta(\mathcal{F})$ as follows: $P \succsim^{\prime} Q$ if

$$
\int_{\mathcal{F}} V(\mathbf{u}(f)) d P \geq \int_{\mathcal{F}} V(\mathbf{u}(f)) d Q \text { for every } V \in \mathbb{V} .
$$

Note that $P \succsim^{\prime} Q$ implies

$$
\begin{aligned}
& \min _{M \in \mathcal{M}} \int_{\mathcal{F}}\left(\min _{\mu \in M} \int_{\mathcal{S}} u(f) d \mu\right) d P \\
= & \int_{\mathcal{F}}\left(\min _{\mu \in M(P)} \int_{\mathcal{S}} u(f) d \mu\right) d P \\
\geq & \int_{\mathcal{F}}\left(\min _{\mu \in M(P)} \int_{\mathcal{S}} u(f) d \mu\right) d Q \\
\geq & \min _{M \in \mathcal{M}} \int_{\mathcal{F}}\left(\min _{\mu \in M} \int_{\mathcal{S}} u(f) d \mu\right) d Q,
\end{aligned}
$$

in which $M(P) \in \mathcal{M}$ is a worst-case scenario for $P$. Therefore, $P \succsim^{\prime} Q \Rightarrow P \succsim Q$. By Proposition 22 of Cerreia-Vioglio (2009), $\widehat{\mathbb{V}} \subset \mathbb{V}$. Since $\phi$ is a bijection, this implies $\widehat{\mathcal{M}} \subset \mathcal{M}$.

Proof of Theorem 4: We only prove the sufficiency of the axioms. Part 1 of Lemma 1 shows that for any lottery $P, P \sim f^{P}$. Therefore, for any DMEU representation of $\succsim$,

$$
W(P)=\min _{M \in \mathcal{M}} \int_{\mathcal{F}}\left(\min _{\mu \in M} \int_{\mathcal{S}} u(f) d \mu\right) d P
$$


it must be true that $W(P)=W\left(f^{P}\right)$. For an act $f$, we know that

$$
W(f)=\min _{\mu \in \mathbb{M}} \int_{\mathcal{S}} u(f) d \mu,
$$

in which $\mathbb{M}=\operatorname{cl}\left(\bigcup_{M \in \mathcal{M}} M\right)$. Therefore,

$$
\begin{aligned}
W(P) & =W\left(f^{P}\right) \\
& =\min _{\mu \in \mathbb{M}} \int_{\mathcal{S}}\left(\int_{\mathcal{F}} u(f) d P\right) d \mu \\
& =\min _{\mu \in \mathbb{M}} \int_{\mathcal{F}}\left(\int_{\mathcal{S}} u(f) d \mu\right) d P .
\end{aligned}
$$

Since $\Delta(\mathcal{S})$ is compact, $\mathbb{M}$ is compact.

Proof of THEOREM 5: We only show that if $\succsim$ has a DMEU representation and satisfies Independence, then it has an Ex Post MEU representation. Since Weak Order, Continuity, and Independence hold, there exists a continuous expected utility function $V^{\prime}: \mathcal{F} \rightarrow \mathbb{R}$ such that

$$
W^{e p}(P)=\int_{\mathcal{F}} V^{\prime}(f) d P
$$

represents $\succsim$.

Define $u(p)=W^{e p}(p)$ for any $p \in \Delta(\mathcal{X})$. In the proof of Theorem 2, we use $x_{l}$ to denote one of the worst lotteries and $x_{h}$ one of the best lotteries. Since FOSD holds, whenever two acts $f$ and $g$ satisfy $W^{e p}\left(f_{i}\right) \geq W^{e p}\left(g_{i}\right)$ for any $i, W^{e p}(f) \geq W^{e p}(g)$. Therefore, there exists a function $V^{*}:\left[u\left(x_{l}\right), u\left(x_{h}\right)\right]^{n} \rightarrow \mathbb{R}$ such that

$$
V^{*}\left(u\left(f_{1}\right), \ldots, u\left(f_{n}\right)\right)=V^{\prime}(f)
$$


for each $f \in \mathcal{F}$. Notice that for any $p \in \Delta(\mathcal{X})$,

$$
V^{*}(u(p), \ldots, u(p))=V^{\prime}(p)=W^{e p}(p)=u(p) .
$$

Futhermore, due to Continuity and Indifference to Mixture Timing of Constant Acts, for any $p \in \Delta(\mathcal{X})$,

$$
W^{e p}(p)=W^{e p}\left(\int_{\mathcal{X}} \delta_{x} d p\right)=\int_{\mathcal{X}} V^{\prime}\left(\delta_{x}\right) d p=\int_{\mathcal{X}} u\left(\delta_{x}\right) d p
$$

in which $W^{e p}(p)$ is the utility of a degenerate lottery that yields the constant act $p$ with certainty, and $W^{e p}\left(\int_{\mathcal{X}} \delta_{x} d p\right)$ is the utility of the randomization over consequences according to $p$. To show that (17) holds, we first verify that it holds when $p$ has finite support, and then we apply Continuity to the case in which $p$ may not have finite support. Since $W$ is continuous, (16) and (17) imply that $u$ is a continuous expected utility representation of $\succsim$ on $\Delta(\mathcal{X})$.

We know that $\succsim$ has a DMEU representation $(\mathcal{M}, v)$. Since both $u$ and $v$ are expected utility representations of $\succsim$ on $\Delta(\mathcal{X}), v$ is equal to some positive affine transformation of

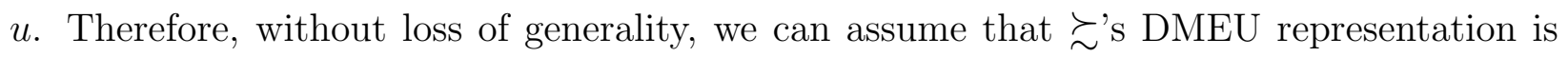
$(\mathcal{M}, u)$; that is,

$$
W(P)=\min _{M \in \mathcal{M}} \int_{\mathcal{F}}\left(\min _{\mu \in M} \int_{\mathcal{S}} u(f) d \mu\right) d P
$$

represents $\succsim$. Note that for any $P, \alpha^{P} x_{h}+\left(1-\alpha^{P}\right) x_{l} \sim P$, as shown in the proof of Lemma 4. We have $W(P)=\alpha^{P} u\left(x_{h}\right)+\left(1-\alpha^{P}\right) u\left(x_{l}\right)=W^{e p}(P)$. In particular, for any $f \in \mathcal{F}$,

$$
W^{e p}(f)=V^{*}(f)=W(f)=\min _{\mu \in \mathbb{M}} \int_{\mathcal{S}} u(f) d \mu,
$$

in which $\mathbb{M}=\operatorname{cl}\left(\bigcup_{M \in \mathcal{M}} M\right)$; that is, $W^{e p}(P)=\int\left(\min _{\mu \in \mathbb{M}} \int_{\mathcal{S}} u(f) d \mu\right) d P$. Since $\Delta(\mathcal{S})$ is compact, $\mathbb{M}$ is compact. 
Comparative Statics: Below is some comparative static result for the DMEU representation. Suppose there are two individuals whose preferences over lotteries are $\succsim_{1}$ and $\succsim_{2}$, respectively. If $\succsim_{i}$ has a DMEU representation $\left(\mathcal{M}_{i}, u_{i}\right)$, we use $\mathbb{M}_{i}$ to denote $\bigcup_{M \in \mathcal{M}_{i}} M$, $i=1,2$. The proposition below provides a sufficient condition for when the individual with $\succsim_{2}$ always thinks that randomization is less effective in eliminating the effect of ambiguity than the individual with $\succsim_{1}$. The necessary condition may also be established, but we will need to focus on DMEU* representations with the unique minimal set of scenarios.

Proposition 1 Suppose $\succsim_{1}$ and $\succsim_{2}$ have DMEU representations $\left(\mathcal{M}_{1}, u\right)$ and $\left(\mathcal{M}_{2}, u\right)$, respectively, and $\mathbb{M}_{1}=\mathbb{M}_{2}$. If for any $M_{1} \in \mathcal{M}_{1}$, there exists an $M_{2} \in \mathcal{M}_{2}$ such that $M_{1} \subset M_{2}$, then $P \succsim_{2} f$ implies $P \succsim_{1} f$ for any lottery $P$ and act $f$.

Proof of Proposition 1: For each lottery $P$, let $M(P)$ denote an element of $\mathcal{M}_{1}$ that minimizes $\int_{\mathcal{F}}\left(\min _{\mu \in M} \int_{\mathcal{S}} u(f) d \mu\right) d P$. For each $M_{1} \in \mathcal{M}_{1}$, let $M_{2}\left(M_{1}\right)$ denote an element of $\mathcal{M}_{2}$ that contains $M_{1}$. Since the two individuals share the same $u$ and $\mathbb{M}_{1}=\mathbb{M}_{2}$, they must also share the same evaluation of every act. Then, the proposition follows from

$$
\begin{aligned}
W_{1}(P) & =\min _{M \in \mathcal{M}_{1}} \int_{\mathcal{F}}\left(\min _{\mu \in M} \int_{\mathcal{S}} u(f) d \mu\right) d P \\
& =\int_{\mathcal{F}}\left(\min _{\mu \in M(P)} \int_{\mathcal{S}} u(f) d \mu\right) d P \\
& \geq \int_{\mathcal{F}}\left(\min _{\mu \in M_{2}(M(P))} \int_{\mathcal{S}} u(f) d \mu\right) d P \\
& \geq \min _{M \in \mathcal{M}_{2}} \int_{\mathcal{F}}\left(\min _{\mu \in M} \int_{\mathcal{S}} u(f) d \mu\right) d P \\
& =W_{2}(P) .
\end{aligned}
$$

